\title{
Gene expression analysis reveals early changes in several molecular pathways in cerebral malaria-susceptible mice versus cerebral malaria-resistant mice
}

\author{
Nicolas F Delahaye ${ }^{1,6}$, Nicolas Coltel ${ }^{2}$, Denis Puthier ${ }^{3}$, Mathieu Barbier ${ }^{1}$, \\ Philippe Benech ${ }^{3}$, Florence Joly ${ }^{3}$, Fuad A Iraqi ${ }^{4}$, Georges E Grau ${ }^{2,5}$, \\ Catherine Nguyen ${ }^{3}$ and Pascal Rihet*1,3
}

Address: ${ }^{1}$ Laboratoire de Pharmacogénétique des maladies parasitaires-EA864, Université de la Méditerranée, IFR48, Marseille, France, ${ }^{2}$ Université de la Méditerranée-IFR48, CNRS-UMR 6020-Immunopathology group, Marseille, France, ${ }^{3}$ INSERM ERM 206-TAGC, Université de la Méditerranée, IFR137, Marseille, France, ${ }^{4}$ Tel-Aviv University, Department of human microbiology, Sackler Faculty of Medicine, Ramat-Aviv, TelAviv 69978, Israel, 5 University of Sydney, Department of Pathology, Faculty of Medicine and Bosch Institute, Australia and ${ }^{6}$ Institut Gustave Roussy, Villejuif, France

Email: Nicolas F Delahaye - nicolasdelahaye@yahoo.fr; Nicolas Coltel - nicolas.coltel@medecine.univ-mrs.fr;

Denis Puthier - puthier@tagc.univ-mrs.fr; Mathieu Barbier - mat.barbier@wanadoo.fr; Philippe Benech - philippe.benech@gensodi.com;

Florence Joly - florence.joly@yahoo.fr; Fuad A Iraqi - fuadi@post.tau.ac.il; Georges E Grau - ggrau@med.usyd.edu.au;

Catherine Nguyen - nguyen@tagc.univ-mrs.fr; Pascal Rihet* - rihet@luminy.univ-mrs.fr

* Corresponding author

Published: 6 December 2007

BMC Genomics 2007, 8:452 doi:10.1/86/147|-2164-8-452
Received: 14 September 2007

Accepted: 6 December 2007

This article is available from: http://www.biomedcentral.com/I47I-2/64/8/452

(c) 2007 Delahaye et al; licensee BioMed Central Ltd.

This is an Open Access article distributed under the terms of the Creative Commons Attribution License (http://creativecommons.org/licenses/by/2.0), which permits unrestricted use, distribution, and reproduction in any medium, provided the original work is properly cited.

\begin{abstract}
Background: Microarray analyses allow the identification and assessment of molecular signatures in whole tissues undergoing pathological processes. To better understand cerebral malaria pathogenesis, we investigated intra-cerebral gene-expression profiles in well-defined genetically cerebral malaria-resistant (CM-R) and CM-susceptible (CM-S) mice, upon infection by Plasmodium berghei ANKA $(\mathrm{PbA})$. We investigated mouse transcriptional responses at early and late stages of infection by use of cDNA microarrays.

Results: Through a rigorous statistical approach with multiple testing corrections, we showed that $\mathrm{PbA}$ significantly altered brain gene expression in CM-R (BALB/C), and in CM-S (CBA/J and C57BL/ 6) mice, and that 327 genes discriminated between early and late infection stages, between mouse strains, and between CM-R and CM-S mice. We further identified 104, 56, 84 genes with significant differential expression between CM-R and CM-S mice on days 2, 5, and 7 respectively. The analysis of their functional annotation indicates that genes involved in metabolic energy pathways, the inflammatory response, and the neuroprotection/neurotoxicity balance play a major role in cerebral malaria pathogenesis. In addition, our data suggest that cerebral malaria and Alzheimer's disease may share some common mechanisms of pathogenesis, as illustrated by the accumulation of $\beta$-amyloid proteins in brains of CM-S mice, but not of CM-R mice.
\end{abstract}

Conclusion: Our microarray analysis highlighted marked changes in several molecular pathways in CM-S compared to CM-R mice, particularly at early stages of infection. This study revealed some promising areas for exploration that may both provide new insight into the knowledge of $C M$ pathogenesis and the development of novel therapeutic strategies. 


\section{Background}

Malaria is a disease affecting millions of people worldwide. Cerebral malaria $(\mathrm{CM})$ is one of the most severe complications and is a major cause of death. Both host and parasite genetic factors play important roles in the outcome of malaria infection. Epidemiological data, candidate gene studies, and genetic linkage studies clearly support the existence of a genetic contribution to susceptibility to human malaria [1]. In parallel with human studies, malaria susceptibility genes have been mapped in mouse models, and the role of some genes has been demonstrated [2]. It is clear, however, that a number of malaria susceptibility genes remain to be identified. These include genes, whose expression is likely deregulated upon malaria infection.

Transcriptional profiling may provide new tools for identifying the key genes that govern host responses against pathogens. Recently, several reports have described gene expression changes that accompany the host response against Plasmodium spp. Microarrays have been analyzed from mice [3-5], rhesus monkey [6] and humans [7], upon infection by Plasmodium spp A parallel can be observed in the regulation of genes involved in immune responses, glycolysis, and erythropoiesis. These data suggested that variation in host gene expression may be associated with resistance or susceptibility to malaria.

Recently, we investigated brain gene expression patterns in well-defined genetically CM-resistant (CM-R) and CMsusceptible mice (CM-S) by use of cDNA microarrray [8]. We identified a set of genes that perfectly discriminates between CM-R and CM-S mice at the time of CM onset. This indicates that gene expression analysis using microarray tools may be useful for the identification of candidate genes that are potentially responsible for resistance or susceptibility to CM. Nevertheless, an important issue was to identify genes whose expression differ between CM-R and CM-S mice before the time of CM onset to identify early events that may participate in malaria pathogenesis. In this report, we present an analysis of genes differentially expressed in brains from CM-R and CM-S mice prior to infection, and at the early and late stages of infection with Plasmodium berghei ANKA (PbA). Data analysis reveals that molecules belonging to several biological processes were preferentially and differentially expressed between CM-R and CM-S mice, and that a number of gene expression changes occurred at the early and late stages of infection. Herein, we discuss new working hypotheses on this basis.

\section{Results \\ Identification of genes regulated in brains by $\mathrm{PbA}$ infection} The ANOVA of microarray data revealed significant gene expression changes over the course of infection in BALB/C $(\mathrm{n}=25)$ mice, CBA/J mice $(\mathrm{n}=16)$, and in C57BL/6 mice $(n=20)$. We calculated empirical $P$ values for each gene, and we considered $P<0.05$ significant. On this basis, we selected 174, 210, and 342 genes for BALB/c mice, CBA/J mice, and C57BL/ 6 mice, respectively.

To further compare uninfected mice with infected mice, we performed a Welch t test and we applied a Bonferroni correction to account for multiple tests performed (Figure $1)$. Figure 2 shows the number of genes whose expression was significantly altered by $\mathrm{PbA}$ infection. The mouse strains displayed various patterns. Strikingly, the number of genes that showed significant expression changes was higher in C57BL/ 6 mice than in CBA/J mice on day 2 postinfection with PbA (Figure 2A). The number of genes that showed significant expression changes in CBA/J mice gradually increased during infection. In contrast, the number of genes that showed significant expression changes in both C57BL/ 6 mice and BALB/c mice on day 2 was similar to the number of genes identified on day 7 ; it was, nevertheless, lower on day 5 than on days 2 and 7 . As shown in Figure 2, most of the genes identified in C57BL/ 6 mice (Figure 2B) and in CBA/J mice (Figure 2C) were under-expressed, while most of the genes identified in BALB/c mice (Figure 2D) were over-expressed.

A Venn diagram summarizes the number of overlapping genes with significant differential expression at different time points (Figure 3). Most of the genes identified on day

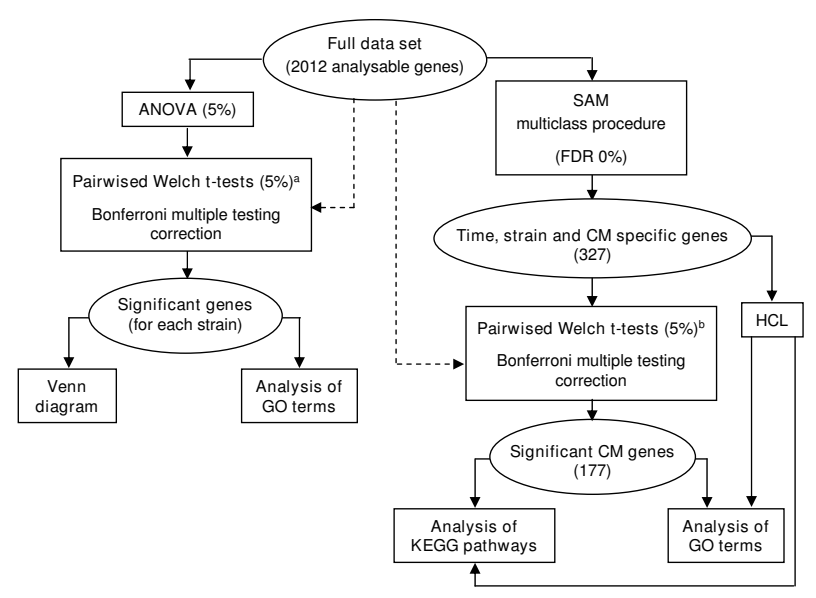

Figure I

Schematic outline of data analysis. HCL: hierarchical clustering. a Brain gene expression prior to infection was compared with brain gene expression on days 2,5 and $7 . b$ Brain gene expression in CM-R mice was compared with that in CM-S mice at each time point. As represented by dashed arrows, we considered the whole data set $(n=2012$ genes $)$ to carry out multiple testing correction. 
A

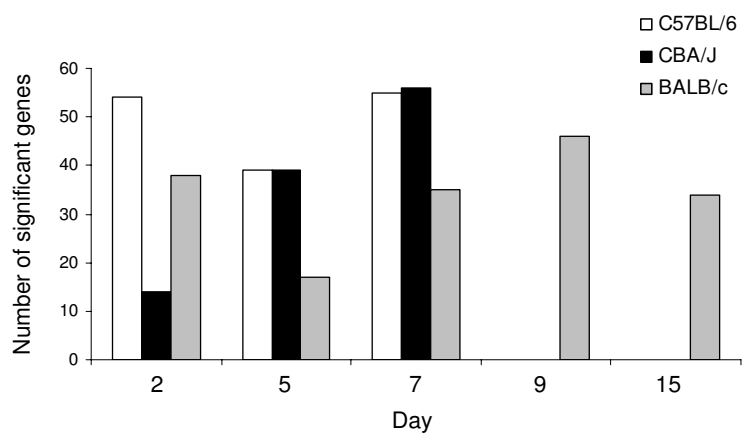

B

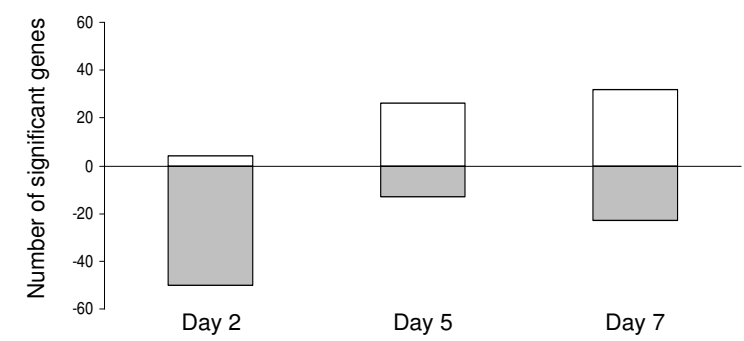

C

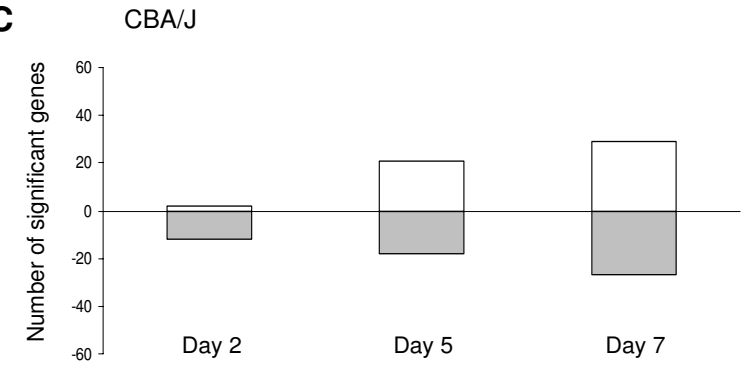

D $B A L B / c$

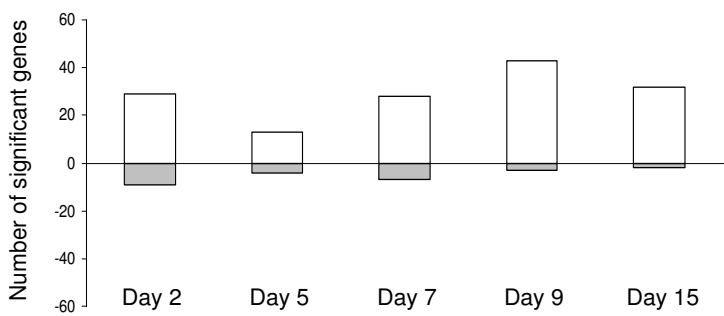

Figure 2

Distribution of genes regulated by $\mathrm{PbA}$ infection according to the time of infection. The genes whose expression was significantly altered by $\mathrm{PbA}$ infection were identified by using pair-wise Welch $t$ tests with a Bonferroni correction.A. The number of genes with significant changes is shown at each time point for each mouse strain. B, C and D. The number of genes significantly up- (positive values) and down-regulated (negative values) is shown at each time point for each mouse strain.
2 were no longer identified on day 7 in C57BL/ 6 mice and in BALB/c mice. As shown in Figure 3A-B and 3E-F, 42 of 53 and 31 of 36 genes with differential expression on day 2 did not show differential expression on days 5 and 7 in $\mathrm{C} 57 \mathrm{BL} / 6$ and $\mathrm{BALB} / \mathrm{c}$ mice, respectively. In contrast, 10 of 14 genes regulated by $\mathrm{PbA}$ infection on day 2 were also regulated on days 5 and 7 in CBA/J mice (Figure 3C-D), indicating that $\mathrm{C} 57 \mathrm{BL} / 6$ mice and $\mathrm{CBA} / \mathrm{J}$ mice partly differ in their transcriptional response over the course of infection.

Microarray analysis discriminates between CM-R and CM$S$ mice according to the time after infection

We searched for genes showing differential expression between mouse strains before infection. The ANOVA identified 125 genes without multiple test correction (data not shown), and 7 genes with Bonferroni multiple
A

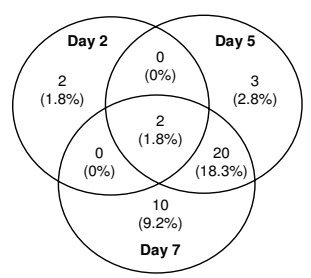

C CBA/J

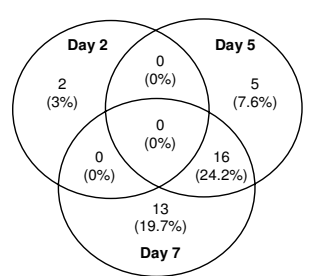

E $B A L B / C$

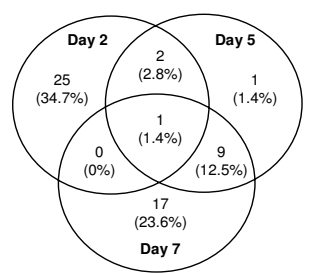

B $\quad$ C57BL/6

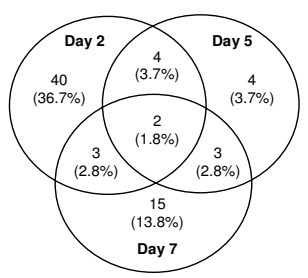

D CBA/J

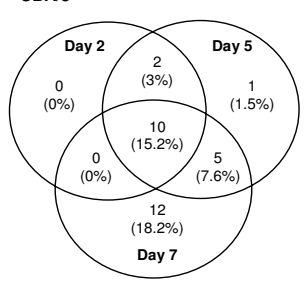

F BALB/C

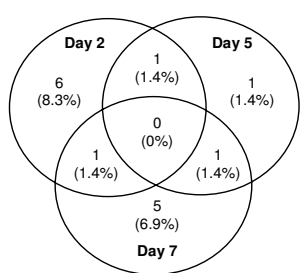

Figure 3

Overlapping genes with significant differential expression at different time points during $\mathrm{PbA}$ infection. This Venn diagrams show the number of overlapping genes with significant expression changes on days 2, 5, and 7 post-infection with $\mathrm{PbA}$, for $\mathrm{C} 57 \mathrm{BL} / 6$ mice (A and $\mathbf{B}), \mathrm{CBA} / \mathrm{J}$ mice ( $\mathbf{C}$ and $\mathbf{D})$ and $B A L B / c$ mice (E and $\mathbf{F})$. Genes that are either induced or suppressed by infection are distinguished for each strain: the number of genes induced is shown in $\mathbf{A}$, $\mathbf{C}$, and $\mathbf{E}$, while the number of genes suppressed is shown in B, D, and F. 
test correction at the level of 5\%: 1700023B02Rik, Acot8, Gpd2, Sec11c, Ngly1, Zfp346 and Tln2. These results revealed a minor natural variation in gene expression between mouse strains. Nevertheless, we took into account this natural variation in further analyses to search for transcriptional changes. Thus, the individual gene expression level in infected mice was adjusted for the gene expression level in uninfected mice for each mouse strain.

To focus on transcriptional changes associated with resistance or susceptibility, we searched for a set of genes that discriminated between CM-R mice (BALB/c) and CM-S mice $(\mathrm{C} 57 \mathrm{BL} / 6$ and $\mathrm{CBA} / \mathrm{J})$. To this end, we used the multi-class Significant Analysis of Microarrays (SAM) procedure, and we applied a false discovery rate of $0 \%$ (Figure 1 ). The analysis yielded a set of 327 genes [see Additional file 1], which was used to perform unsupervised hierarchical clustering (Figure 4A). Interestingly, the expression of several genes was induced in CM-R mice or suppressed in CM-S mice as a result of infection, while the expression of other genes was suppressed in CM-R mice and induced in CM-S mice [see Additional file 1]. This led to successfully classify CM-R and CM-S mice according to the time after infection (Figure 4B) and to determine five clusters (Figure $4 \mathrm{~A})$. Clusters $\mathrm{A}$ and $\mathrm{C}$ fully grouped $\mathrm{CM}-\mathrm{R}$ mice (BALB/c) versus CM-S mice (C57BL/6 and CBA/J) whatever the time after infection. Genes of cluster A were overexpressed in CM-S mice compared to CM-R mice, while genes of cluster $\mathrm{C}$ were under-expressed in CM-S mice compared to CM-R mice. Clusters B and D also grouped CM-R mice versus CM-S mice. Nevertheless, clusters B and $\mathrm{D}$ discriminated between $\mathrm{C} 57 \mathrm{BL} / 6$ and $\mathrm{CBA} / \mathrm{J}$ mice, indicating differential gene expression between the two CM-S strains at early time points of infection. Genes of clusters $\mathrm{B}$ and $\mathrm{D}$ were over-expressed in CBA/J mice on day 2 postinfection compared to C57BL/6 mice, and in C57BL/6 on days 2 and 5 post-infection compared to CBA/J mice, respectively. At late stage of infection, genes of clusters $B$ and $\mathrm{D}$ showed similar gene expression between the two CM-S strains, and were under-expressed in CM-S mice compared to CM-R mice. Cluster E discriminated mice at early and late stage of infection. Nevertheless, genes of cluster E were over-expressed in CM-S mice compared to CM-R mice indicating an interval between the response of $\mathrm{CM}-\mathrm{S}$ and the response of CM-R mice.

To analyze functional annotations related to $\mathrm{CM}$, we sought biological process Gene Ontology (GO) terms and KEGG pathways for the 327 genes that discriminated between CM-R mice (BALB/c) and CM-S mice (C57BL/6 and CBA/J). The analysis of biological process GO terms of gene clusters showed an over-representation of some $\mathrm{GO}$ terms. In particular, the GO terms related to the "defense response" category, such as the "immune response" or the "inflammatory response" GO terms were strongly over-represented in cluster E (Table 1). Similarly, most of the genes grouped in cluster E were found to be involved in KEGG pathways related to immune responses, such as "cytokine-cytokine receptor interaction" or "natural killer cell mediated cytotoxicity". The analysis of other clusters did not reveal, however, a strong over-representation of particular GO terms, suggesting that these clusters were heterogeneous. In particular, clusters $\mathrm{B}$ and $\mathrm{C}$ contained 38 and 207 genes with heterogeneous GO categories. This was further supported by the analysis of KEGG pathways, which pointed out a number of different pathways, such as metabolic energy pathways ("glycolysis/gluconeogenesis" and "oxidative phosphorylation"), immune responses ("cytokine-cytokine receptor interaction" and "antigen processing and presentation"), haematopoiesis, ("hematopoietic cell lineage"), cytoskeleton pathways ("regulation of active cytoskeleton"), or pathways related to brain function ("neurodegenerative disorders" and "axon guidance") (Figure 5).

We specifically searched for genes with significant differential expression between CM-R and CM-S mice. Thus, we used the Welch t test to compare BALB/c mice (CM-R mice) on the one hand and CBA/J mice and C57BL/6 mice (CM-S mice) on the other hand (Figure 1). We tested the 327 genes identified by the SAM multi-class procedure, but we considered the whole data set $(n=2012$ genes $)$ to carry out multiple testing correction. Among the 327 genes, 177 genes were further found to be differentially expressed between CM-R mice and CM-S mice (Table 2). Among the 177 genes, 104, 56, and 84 genes showed significant differential expression on days 2, 5, and 7, respectively, and 11,17,138, and 11 were grouped in clusters $A$, $\mathrm{B}, \mathrm{C}$ and $\mathrm{E}$, respectively. The number of co-occurring genes with differential expression between CM-R and CM-S mice detected at different times is displayed in Figure 6. Eighteen of 177 genes were up-regulated in CM-S mice compared to CM-R mice, while 158 of 177 genes were upregulated in CM-R mice compared to CM-S mice. Only one gene showed a complicated picture: Fyco1 was downregulated in $\mathrm{CBA} / \mathrm{J}$ and $\mathrm{C} 57 \mathrm{BL} / 6$ mice (CM-S mice) mice on day 2, and in CBA/J mice on day 5, while it was up-regulated in $\mathrm{C} 57 \mathrm{BL} / 6$ mice on day 5 . Table 3 show the most represented KEGG pathways, which included pathways related to metabolism, erythropoiesis, immune responses, neuronal development, and neurodegenerative disorders.

We further analyzed the expression of genes involved in neuronal development or in neurodegenerative disorders using immunochemistry. Thus, we studied the expression of reelin (Reln), which is involved in neurogenesis, and we searched for the presence of $\beta$-amyloid protein, which is involved in Alzheimer's disease. We detected RELN only in brains of BALB/C mice at days 5 and 7, while we showed 
A

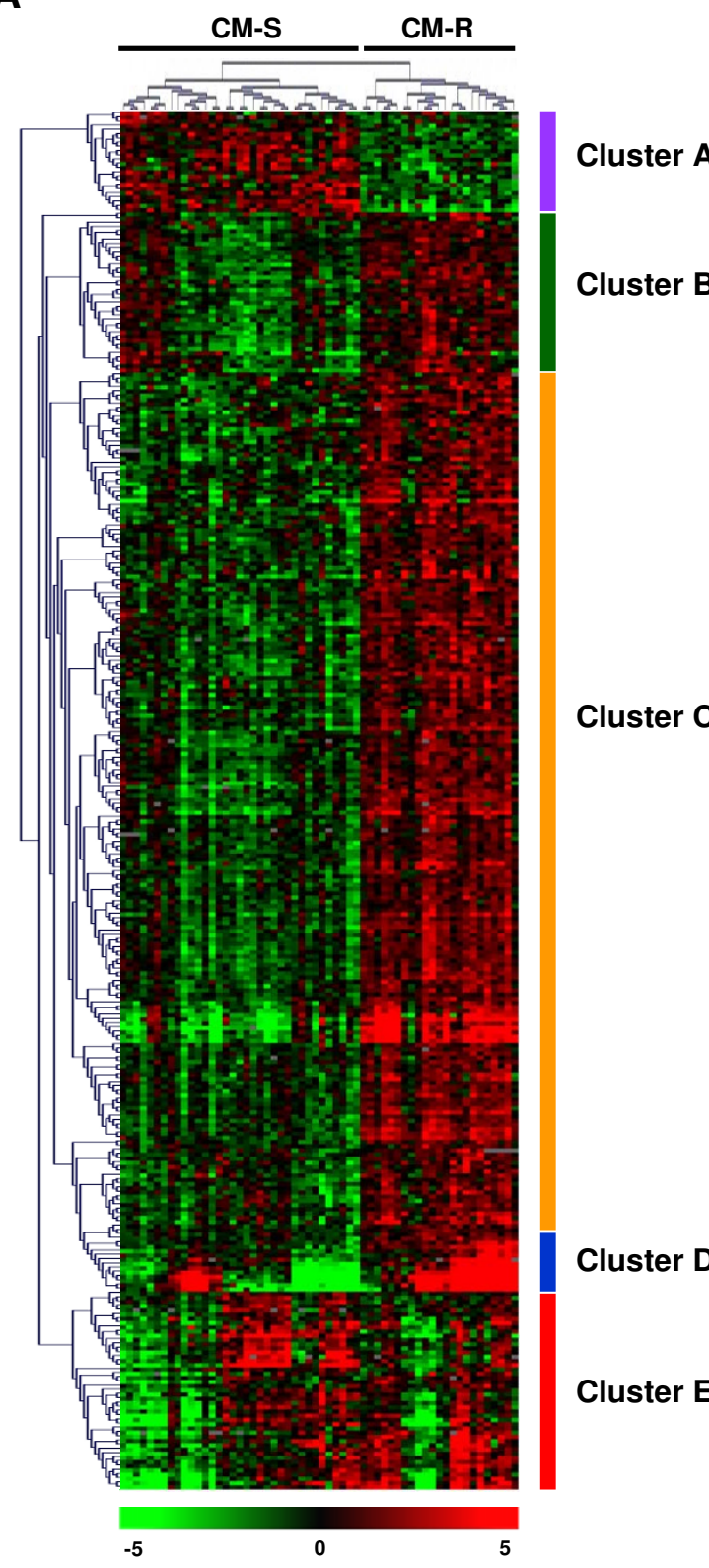

B

mice

Early

time

points

mice

Late

time

points

CM-R

mice

Early and late time points

D9-15

D7

D7
CBA/J-5 replicate1 D2

CBA/J-5 replicate2 D2

CBA/J-5 replicate3 D2

CBA/J-5 replicate4 D2

CBA/J-5 r rA/J-4 D2 eplicate5 D2 C57BL6-8 D5 C57BL6-11 D5 C57BL6-4 D2 C57BL6-4 D2 C57BL6-6 D2 C57BL6-7 D2 C57BL6-10 D5 C57BL6-5 D2 C57BL6-9 D5 C57BL6-16 D7 C57BL6-17 D7 C57BL6-12 D7 C57BL6-18 D7

C57BL6-20 replicate1 D7 C57BL6-13 D7 C57BL6-19 D7 C57BL6-14 D7 C57BL6-15 D7 C57BL6-20 replicate2 D7 CBA/J-10 D5

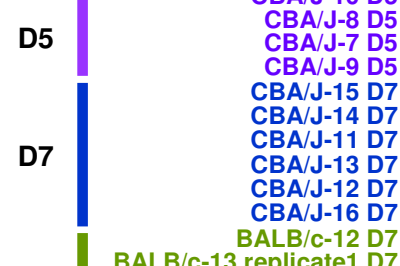

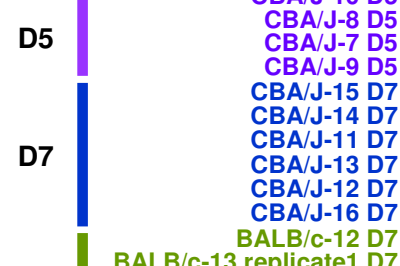

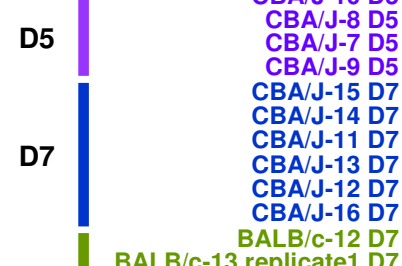

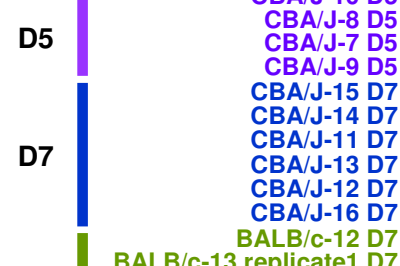

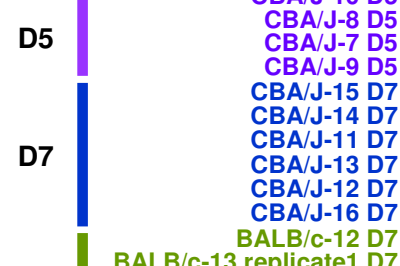

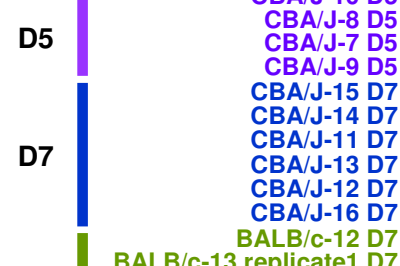

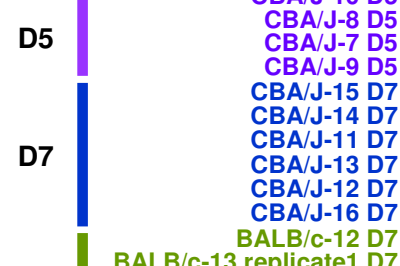

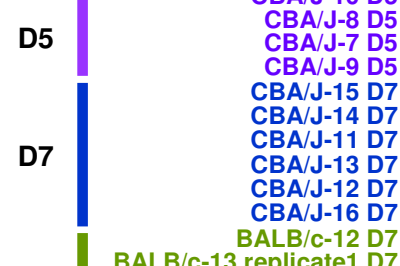

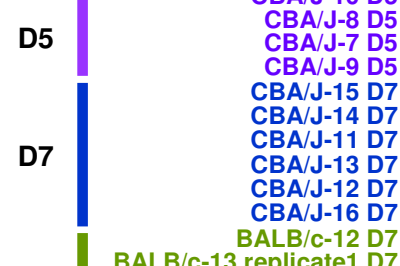

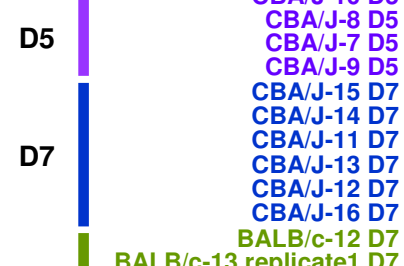

BALB/C-13 replicate2 D7 BALB/c-11 D7 BALB/c-14 D7 BALB/C-15 D7 BALB/C-10 D5 $B A L B / C-4$ D2 $\mathrm{BALB} / \mathrm{C}-5 \mathrm{D} 2$ BALB/C-6 2 $\mathrm{BALB} / \mathrm{C}-8$ D5 BALB/C-8 55 BALB/c-17 D9 BALB/C-17 D9 BALB/C-20 D9 BALB/C-22 D15 BALB/C-18 D9 BALB/C-19 D9 BALB/C-23 D15 BALB/C-21 D15 BALB/C-24 D15 BALB/C-25 D15

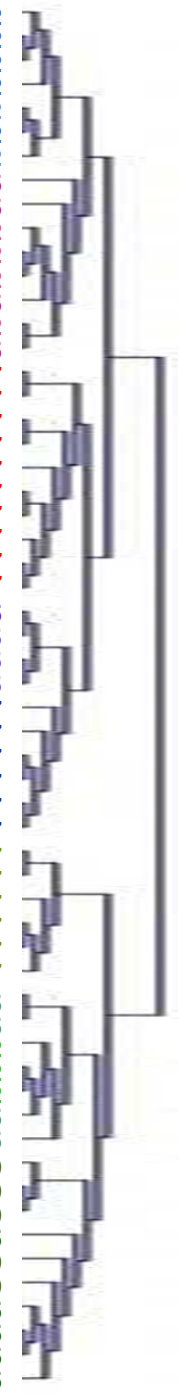

\section{Figure 4}

Hierarchical classification of mouse-strain-specific and CM-R/CM-S genes according to the time of infection. A. Hierarchical clustering of the 58 brain tissue samples, using expression levels of 327 significant genes differentially expressed between the mouse strains at early and late stages of infection. This set of genes was extracted from the full data set $(n=2012)$ by use of a SAM procedure and a false discovery rate of $0 \%$. Each row represents a gene and each column represents a sample. Red and green indicate expression levels above and below the median, respectively. Grey indicates missing data. Dendograms of samples (above matrix) and genes (to the left of matrix) represent overall similarities in gene expression profiles. B. Dendogram of samples representing the results of the same global hierarchical clustering applied to the 58 brain tissue samples. Clustering of technical replicates (CBA/J-5, C57BL6-20 and BALB/c-13) is shown: the samples taken from a CBA/J mouse on day 2, a C57L/6 mouse on day 7 , and a BALB/c mouse on day 7 were run on 5,2 , and 2 microarrays, respectively. Samples taken from mice on days $2,5,7,9$, and I5 post-infection are coded D2, D5, D7, D9, and DI5, respectively. 
Table I: Enrichment of the 327 genes differentially expressed $^{\mathrm{a}}$ in Gene Ontology terms

\begin{tabular}{|c|c|}
\hline Cluster, GO term & Fisher exact test $t^{b}$ \\
\hline \multicolumn{2}{|l|}{ Cluster A } \\
\hline Defense response ${ }^{c}$ & 0.043 \\
\hline \multicolumn{2}{|l|}{ Cluster B } \\
\hline$\ldots$ & $\ldots$ \\
\hline \multicolumn{2}{|l|}{ Cluster C } \\
\hline Positive regulation of cell activationc & 0.0024 \\
\hline $\begin{array}{l}\text { Positive regulation of lymphocyte } \\
\text { activationc }\end{array}$ & 0.0024 \\
\hline \multicolumn{2}{|l|}{ physiological processc } \\
\hline Regulation of phosphorylationc & 0.008 \\
\hline Cell death & 0.013 \\
\hline Positive regulation of immune response & 0.015 \\
\hline $\begin{array}{l}\text { Positive regulation of cellular } \\
\text { biosynthesis }\end{array}$ & 0.036 \\
\hline Cytokines and inflammatory response & 0.049 \\
\hline \multicolumn{2}{|l|}{ Cluster D } \\
\hline$\ldots$ & $\ldots$ \\
\hline \multicolumn{2}{|l|}{ Cluster E } \\
\hline Defense response & $0.000000 \mathrm{Id}^{\mathrm{d}}$ \\
\hline Immune response & $0.000004^{d}$ \\
\hline Response to pest/pathogen/parasite & $0.000005 I^{d}$ \\
\hline Inflammatory response & 0.0027 \\
\hline Response to stress & 0.0028 \\
\hline Response to wounding & 0.003 \\
\hline Chemotaxis & 0.0054 \\
\hline Response to external stimulus & 0.011 \\
\hline
\end{tabular}

a This set of genes was extracted from the full data set $(n=2012)$ by use of a SAM procedure and a false discovery rate of $0 \%$.

b Significant results with a Fischer exact test $(P<0.05)$.

c GO terms that were also over-represented in the set of 177 significant $\mathrm{CM}$ genes.

d Significant results with the Benjamini multi-testing correction $(\mathrm{P}<$ $0.05)$.

the presence of $\beta$-amyloid only in brains of $\mathrm{CBA} / \mathrm{J}$ and C57BL/6 mice at days 5 and 7 (Table 4).

Overall, our gene expression analysis revealed marked changes in metabolic energy pathways, the inflammatory response, and pathways related to neurogenesis and neurodegenerative disorders in CM-S mice versus CM-R mice.

\section{Discussion}

In the present study, we have searched for genes and physiological pathways potentially involved in $\mathrm{CM}$. To this aim, we performed a longitudinal analysis of differentially expressed genes in brains from well-defined genetically $\mathrm{CM}-\mathrm{R}(\mathrm{BALB} / \mathrm{C})$ and $\mathrm{CM}-\mathrm{S}(\mathrm{C} 57 \mathrm{BL} / 6$, and $\mathrm{CBA} / \mathrm{J})$ mice at early and late stages of infection. The present study shows that $\mathrm{PbA}$ strongly altered gene expression in these mice. In particular, gene expression was deeply altered at the time of CM onset, confirming our previous study [8]. Here, we show that a number of genes were over-expressed in CM-

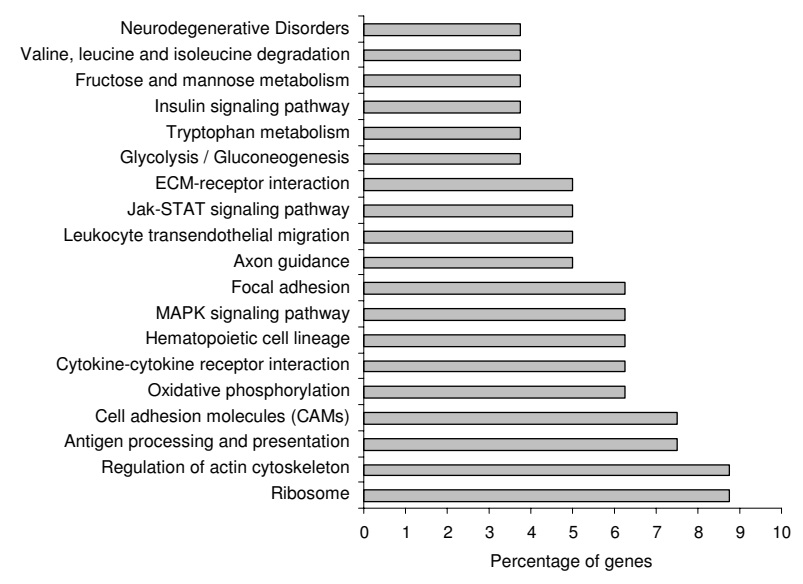

Figure 5

View of biological functional annotation repartition of the genes grouped in clusters $\mathbf{B}$ and $\mathbf{C}$. The KEGG pathways, in which the genes were known to be involved, are shown. Of the 245 genes of the clusters B and C, 80 were annotated. We represented only the KEGG pathways that contained at least three genes.

$\mathrm{R}$ mice at the early stage of infection, suggesting that CM$\mathrm{R}$ mice mount an early protective transcriptional response. In this way, we found an association of resistance to $\mathrm{CM}$ with an increase in the expression of a number of genes on day 2 post-infection (Figure 4 and Table 2). In contrast, a number of genes were found to be underexpressed in C57BL/6 mice at the early stage of infection, while few genes were found to be regulated in $\mathrm{CBA} / \mathrm{J}$ mice at the same time (Figure 2). Also, CBA/J mice appeared to mount a gradual response that may be involved in $\mathrm{CM}$ pathogenesis, while C57BL/ 6 mice may mount two waves of transcriptional responses both of them potentially implicated in malaria pathogenesis. These observations are consistent with other reports suggesting that $\mathrm{CM}$ mediators partly differ between $\mathrm{C} 57 \mathrm{BL} / 6$ and $\mathrm{CBA} / \mathrm{J}$ mice [810]. Nevertheless, both CBA/J and C57BL/ 6 mice showed a pronounced up-regulation of genes involved in either interferon-associated response or in glycolysis at the late stage, and a down-regulation of genes involved in erythropoiesis both at early and late stages, as previously described [3].

To identify genes with significant transcriptional changes associated with $\mathrm{CM}$, we performed, on the whole data set, a multi-class SAM procedure with a very stringent false discovery rate followed by a Welch $\mathrm{t}$ test with a Bonferroni correction. In other words, we applied a two-filtering procedure to decrease the number of "false positive" genes. To account for natural variation between mouse strains, we adjusted gene expression level in infected mice for gene expression level in uninfected mice. Thus, we identi- 
Table 2: CM-specific genes obtained by use of the Welch $t$ test and a Bonferroni correction

\begin{tabular}{|c|c|c|c|c|c|c|c|}
\hline IDa & Cluster & Symbolb & Name & $\mathrm{Chr}^{\mathrm{c}}$ & Welc & test $^{d}$ & \\
\hline BG083931 & A & Slc25all & Solute carrier family 25 (mitochondrial carrier; oxoglutarate carrier), member II & 11 & D2 & $\ldots$ & $\ldots$ \\
\hline 718084 & A & Dfy & Duffy blood group & I & D2 & $\ldots$ & $\ldots$ \\
\hline BG0647|4 & A & Slc3a2 & Solute carrier family 3 (activators of dibasic and neutral amino acid transport), member 2 & 19 & D2 & $\ldots$ & $\ldots$ \\
\hline 573549 & A & Mrps34 & Mitochondrial ribosomal protein S34 & 17 & D2 & D5 & $\ldots$ \\
\hline 641184 & A & Cdc37II & Cell division cycle 37 homolog (S. cerevisiae)-like & 19 & D2 & $\mathrm{D} 5 \mathrm{e}$ & $\ldots$ \\
\hline 641058 & A & Nfkbia & Nuclear factor of kappa light chain gene enhancer in B-cells inhibitor, alpha & 12 & $\ldots$ & D5 & D7 \\
\hline BG086014 & A & HmgnI & High mobility group nucleosomal binding domain I & 16 & D2 & D5 & $\ldots$ \\
\hline$\underline{639606}$ & A & E03004IM2IRik & RIKEN cDNA E03004IM2I gene & 10 & $D 2^{f}$ & D5 & $\ldots$ \\
\hline$\underline{636468}$ & A & Epb4.113 & Erythrocyte protein band 4.I-like 3 & 17 & $\ldots$ & D5 & $\ldots$ \\
\hline BG084377 & A & Stat3 & Signal transducer and activator of transcription 3 & 11 & D2 & $\ldots$ & $\ldots$ \\
\hline$\underline{582660}$ & A & Tagap I & T-cell activation GTPase activating protein I & 17 & D2 & D5 & D7 \\
\hline$\underline{573243}$ & B & Ddi2 & DNA-damage inducible protein 2 & 4 & $\ldots$ & D5 & $\ldots$ \\
\hline BG07II64 & B & 23 I0008MIORik & RIKEN cDNA $2310008 \mathrm{MI} 0$ gene & 3 & D2 & $\ldots$ & D7 \\
\hline BG070859 & B & Nipbl & Nipped-B homolog (Drosophila) & 15 & $\ldots$ & $\mathrm{D} 5 \mathrm{e}$ & D7 \\
\hline$\underline{639002}$ & B & Mlx & MAX-like protein $X$ & 11 & D2 & D5 & D7 \\
\hline BG072955 & B & Мyo5a & Myosin $\mathrm{Va}$ & 9 & $\ldots$ & $\ldots$ & D7 \\
\hline$\underline{575433}$ & B & Ptgerl & Prostaglandin E receptor I (subtype EPI), 42 kD & 8 & $\ldots$ & $\ldots$ & D7 \\
\hline BG087138 & B & Itsn2 & Intersectin 2 & 12 & D2 & $\ldots$ & D7 \\
\hline BG078795 & B & Hspa5 & Heat shock 70 kD protein 5 (glucose-regulated protein) & 2 & $\ldots$ & $\ldots$ & D7 \\
\hline BG064608 & B & Calr & Calreticulin & 8 & $\ldots$ & $\ldots$ & D7 \\
\hline$\overline{B G 087169}$ & B & P4hb & Protein disulfide isomerase associated 6 & 12 & $\ldots$ & $\ldots$ & D7 \\
\hline$\underline{764825}$ & B & Reln & Reelin & 5 & D2 & $\ldots$ & $\ldots$ \\
\hline 1263789 & B & Cxcll2 & Chemokine (C-X-C motif) ligand 12 & 6 & D2 & $\ldots$ & $\ldots$ \\
\hline 441212 & B & Atp6vlg2 & ATPase, $\mathrm{H}+$ transporting, lysosomal $13 \mathrm{kD}, \mathrm{VI}$ subunit $\mathrm{G}$ isoform 2 & 17 & D2 & $\ldots$ & $\ldots$ \\
\hline$\underline{577991}$ & B & Zdhhc6 & Zinc finger, DHHC domain containing 6 & 19 & $\ldots$ & D5 & $\ldots$ \\
\hline$\underline{1263101}$ & B & Hmgcs I & 3-hydroxy-3-methylglutaryl-Coenzyme A synthase I & 13 & $\ldots$ & D5 & $\ldots$ \\
\hline$\underline{573257}$ & B & Pcgf3 & Polycomb group ring finger 3 & 5 & $\ldots$ & $\ldots$ & D7 \\
\hline$\underline{436894}$ & B & Fabp7 & Fatty acid binding protein 7 , brain & 10 & $\ldots$ & $\ldots$ & D7 \\
\hline BG076809 & $\mathrm{C}$ & Rps 18 & Ribosomal protein SI8 & 17 & D2 & $\ldots$ & $\ldots$ \\
\hline BG085025 & $\mathrm{C}$ & Dabl & Disabled homolog I (Drosophila) & 4 & $\ldots$ & D5 & $\ldots$ \\
\hline 718873 & $\mathrm{C}$ & SI00alo & SI00 calcium binding protein AIO (calpactin) & 3 & D2 & D5 & $\ldots$ \\
\hline$\underline{576647}$ & $\mathrm{C}$ & Chchdl & Coiled-coil-helix-coiled-coil-helix domain containing I & 14 & D2 & D5 & $\ldots$ \\
\hline BG063799 & $\mathrm{C}$ & Ak3II & Adenylate kinase 3 alpha-like I & 4 & $\ldots$ & $\ldots$ & D7 \\
\hline BG077012 & $\mathrm{C}$ & Cdc6 & Cell division cycle 6 homolog (S. cerevisiae) & 11 & D2 & D5 & $\ldots$ \\
\hline BG077054 & $\mathrm{C}$ & Ncbp2 & Nuclear cap binding protein subunit $2,20 \mathrm{kDa}$ & 16 & D2 & $\ldots$ & $\ldots$ \\
\hline BG062969 & $\mathrm{C}$ & Hnrpdl & Heterogeneous nuclear ribonucleoprotein D-like & 5 & D2 & $\ldots$ & $\ldots$ \\
\hline 576635 & $\mathrm{C}$ & Vcam I & Vascular cell adhesion molecule I & 3 & D2 & $\ldots$ & $\ldots$ \\
\hline$\underline{402711}$ & $\mathrm{C}$ & Crybbl & Crystallin, beta $\mathrm{BI}$ & 5 & $\ldots$ & $\ldots$ & D7 \\
\hline BG080965 & $\mathrm{C}$ & Prkcbpl & Protein kinase $\mathrm{C}$ binding protein I & 2 & $\ldots$ & $\ldots$ & D7 \\
\hline BG074422 & $\mathrm{C}$ & ltgbl & Integrin beta I (fibronectin receptor beta) & 8 & $\ldots$ & D5 & $\ldots$ \\
\hline BG084605 & $\mathrm{C}$ & Bpgm & 2,3-bisphosphoglycerate mutase & 6 & D2 & $\ldots$ & $\ldots$ \\
\hline BG078316 & $\mathrm{C}$ & Sfxnl & Sideroflexin I & 13 & $\ldots$ & $\ldots$ & D7 \\
\hline BG07606I & $\mathrm{C}$ & Lyar & LyI antibody reactive clone & 5 & $\ldots$ & $\ldots$ & D7 \\
\hline BG077320 & $\mathrm{C}$ & Mrps I0 & Mitochondrial ribosomal protein SIO & 17 & D2 & $\ldots$ & $\ldots$ \\
\hline BG077670 & $\mathrm{C}$ & Gnai2 & Guanine nucleotide binding protein, alpha inhibiting 2 & 9 & D2 & $\ldots$ & D7 \\
\hline$\underline{406838}$ & $\mathrm{C}$ & Foxo3a & Forkhead box O3a & 10 & $\ldots$ & $\ldots$ & D7 \\
\hline BG072299 & $\mathrm{C}$ & Ramp2 & Receptor (calcitonin) activity modifying protein 2 & 19 & $\ldots$ & $\ldots$ & D7 \\
\hline 1224916 & $\mathrm{C}$ & Atxn7 & Ataxin 7 & 14 & D2 & $\ldots$ & $\ldots$ \\
\hline$\overline{1395654}$ & $\mathrm{C}$ & Batf & Basic leucine zipper transcription factor, ATF-like & 12 & D2 & D5 & $\ldots$ \\
\hline BG087349 & $\mathrm{C}$ & Aldh Ibl & Aldehyde dehydrogenase I family, member BI & 4 & D2 & $\ldots$ & $\ldots$ \\
\hline 596637 & $\mathrm{C}$ & Stab2 & Stabilin 2 & 10 & D2 & $\ldots$ & $\ldots$ \\
\hline$\underline{596064}$ & $\mathrm{C}$ & $\mathrm{Cd5}$ & CD5 antigen & 19 & D2 & $\ldots$ & $\ldots$ \\
\hline 573880 & $\mathrm{C}$ & Nfyc & Nuclear transcription factor-Y gamma & 4 & D2 & $\ldots$ & $\ldots$ \\
\hline 1226020 & $\mathrm{C}$ & Unknown & Unknown & $\ldots$ & D2 & $\ldots$ & D7 \\
\hline$\underline{400157}$ & $\mathrm{C}$ & Uqcrq & Ubiquinol-cytochrome $\mathrm{c}$ reductase binding protein & 11 & $\ldots$ & $\ldots$ & D7 \\
\hline BG086406 & $\mathrm{C}$ & Agtr2 & Angiotensin II receptor, type 2 & $x$ & $\ldots$ & $\ldots$ & D7 \\
\hline$\underline{582804}$ & $\mathrm{C}$ & CysI & Cystin I & 12 & $\ldots$ & D5 & $\ldots$ \\
\hline$\underline{576480}$ & $\mathrm{C}$ & $\lg 2 \mathrm{bp} 3$ & Insulin-like growth factor 2 mRNA binding protein 3 & 6 & D2 & D5 & $\ldots$ \\
\hline$\underline{464586}$ & $\mathrm{C}$ & Srr & Serine racemase & 11 & D2 & $\ldots$ & $\ldots$ \\
\hline 575610 & $\mathrm{C}$ & 2700085E05Rik & RIKEN cDNA 2700085E05 gene & II & D2 & $\ldots$ & $\ldots$ \\
\hline 575040 & $\mathrm{C}$ & Sellh & Sell (suppressor of lin-12) I homolog (C. elegans) & 12 & D2 & $\ldots$ & $\ldots$ \\
\hline$\underline{B G 087165}$ & $\mathrm{C}$ & Herc4 & Hect domain and RLD 4 & 10 & D2 & $\ldots$ & D7 \\
\hline BG073254 & $\mathrm{C}$ & Rbmxrt & RNA binding motif protein, $X$ chromosome retrogene & 8 & D2 & $\ldots$ & $\ldots$ \\
\hline$\overline{\mathrm{BG} 073981}$ & $\mathrm{C}$ & Gtpbp8 & GTP-binding protein 8 (putative) & 16 & $\ldots$ & D5 & D7 \\
\hline$\underline{573285}$ & $\mathrm{C}$ & Lrriq2 & Leucine-rich repeats and IQ motif containing 2 & 16 & D2 & $\ldots$ & $\ldots$ \\
\hline$\underline{372621}$ & $\mathrm{C}$ & Icam5 & Intercellular adhesion molecule 5 , telencephalin & 9 & $\ldots$ & $\ldots$ & D7 \\
\hline 1428894 & $\mathrm{C}$ & Ptpns I & Protein tyrosine phosphatase, non-receptor type substrate I & 2 & $\ldots$ & $\ldots$ & D7 \\
\hline BG076772 & $\mathrm{C}$ & Gpd2 & Glycerol phosphate dehydrogenase 2, mitochondrial & 2 & $\ldots$ & $\ldots$ & D7 \\
\hline
\end{tabular}


Table 2: CM-specific genes obtained by use of the Welch $\mathbf{t}$ test and a Bonferroni correction (Continued)

\begin{tabular}{|c|c|c|c|c|c|c|c|}
\hline 752149 & $\mathrm{C}$ & Stk16 & Serine/threonine kinase 16 & $\mathrm{~T}$ & $\ldots$ & D5 & D7 \\
\hline$\underline{573328}$ & C & Thrsp & Thyroid hormone responsive SPOTI4 homolog (Rattus) & 7 & $\ldots$ & D5 & D7 \\
\hline BG087431 & C & $\operatorname{Sec} 31 \mathrm{a}$ & SEC3I homolog A (S. cerevisiae) & 5 & D2 & $\ldots$ & $\ldots$ \\
\hline BG08740I & C & Brd7 & Bromodomain-containing 7 & 8 & D2 & D5 & D7 \\
\hline$\underline{1361813}$ & C & Mmachc & Methylmalonic aciduria cblC type, with homocystinuria & 4 & D2 & $\ldots$ & $\ldots$ \\
\hline BG076501 & C & Sfrs6 & Splicing factor, arginine/serine-rich 6 & 2 & $\ldots$ & $\ldots$ & D7 \\
\hline$\underline{1243669}$ & C & Aldoa & Aldolase I, A isoform & 7 & $\ldots$ & D5 & $\ldots$ \\
\hline$\underline{476643}$ & $\mathrm{C}$ & Sez6l & Seizure related 6 homolog like & 5 & D2 & $\ldots$ & $\ldots$ \\
\hline BG075757 & C & $\mathrm{Cd} 8 \mathrm{I}$ & CD 81 antigen & 7 & D2 & $\ldots$ & $\ldots$ \\
\hline$\underline{574496}$ & C & Uspl9 & Ubiquitin specific peptidase 19 & 9 & D2 & $D 5^{f}$ & $\ldots$ \\
\hline BG065688 & C & Uba52 & Ubiquitin A-52 residue ribosomal protein fusion product I & 8 & $\ldots$ & $\ldots$ & D7 \\
\hline$\underline{B G 078688}$ & $\mathrm{C}$ & Dtnb & Dystrobrevin, beta & 12 & $\ldots$ & $\ldots$ & D7 \\
\hline BG086001 & $\mathrm{C}$ & Sv2a & Synaptic vesicle glycoprotein 2 a & 3 & $\ldots$ & $\ldots$ & D7 \\
\hline BG073641 & $\mathrm{C}$ & Rpl27 & Ribosomal protein L27 & 11 & D2 & $\ldots$ & $\ldots$ \\
\hline BG087402 & $\mathrm{C}$ & Hnrpu & Heterogeneous nuclear ribonucleoprotein $U$ & 1 & $\ldots$ & $\ldots$ & D7 \\
\hline$\underline{595925}$ & C & Sell & Selectin, lymphocyte & 1 & D2 & $\ldots$ & $\ldots$ \\
\hline 1345776 & C & $\mathrm{Rbm} / 4$ & RNA binding motif protein 14 & 19 & $\ldots$ & $\ldots$ & D7 \\
\hline BG077327 & C & Gins4 & GINS complex subunit 4 (SId5 homolog) & 8 & $\mathrm{D} 2$ & D5 & $\ldots$ \\
\hline$\underline{387319}$ & C & Zfp346 & Zinc finger protein 346 & 13 & $\mathrm{D} 2 \mathrm{e}^{\mathrm{e}}$ & $\ldots$ & D7 \\
\hline$\frac{\mathrm{AW} 54656}{\underline{5}}$ & C & Exosc7 & Exosome component 7 & 9 & $\ldots$ & $\ldots$ & D7 \\
\hline BG072456 & C & PihldI & PIHI domain containing I & 7 & $\ldots$ & $\ldots$ & D7 \\
\hline BG074521 & C & Npml & Nucleophosmin I & II & D2 & $\ldots$ & $\ldots$ \\
\hline BG085278 & $\mathrm{C}$ & Rpl22 & Ribosomal protein L22 & 4 & $\mathrm{D} 2 \mathrm{e}^{\mathrm{e}}$ & D5 & D7 \\
\hline$\underline{1445843}$ & C & Ahcyll & S-adenosylhomocysteine hydrolase-like I & 3 & D2 & $\ldots$ & D7 \\
\hline BG087592 & $\mathrm{C}$ & Fbxw7 & F-box and WD-40 domain protein 7, archipelago homolog (Drosophila) & 3 & D2 & D5 & D7 \\
\hline BG064589 & C & Atp6rlal & ATPase, $\mathrm{H}+$ transporting, lysosomal $70 \mathrm{kD}, \mathrm{VI}$ subunit $\mathrm{A}$, isoform I & 16 & D2 & $\ldots$ & D7 \\
\hline$\underline{441227}$ & $\mathrm{C}$ & Abtbl & Ankyrin repeat and BTB (POZ) domain containing I & 6 & D2 & D5 & $\ldots$ \\
\hline$\underline{386911}$ & C & Trim29 & Tripartite motif protein 29 & 9 & $\ldots$ & $\ldots$ & D7 \\
\hline$\underline{B G 087083}$ & C & Serinc3 & Serine incorporator 3 & 2 & D2 & $\ldots$ & $\ldots$ \\
\hline$\underline{372340}$ & C & Myod I & Myogenic differentiation I & 7 & D2 & $\ldots$ & $\ldots$ \\
\hline$\underline{B G 081915}$ & C & Ube3a & Ubiquitin protein ligase E3A & 7 & D2 & D5 & $\ldots$ \\
\hline$\underline{573124}$ & C & Mrrf & Mitochondrial ribosome recycling factor & 2 & D2 & D5 & $\ldots$ \\
\hline$\underline{573230}$ & C & 4930455C2I Rik & RIKEN cDNA 4930455C2I gene & 16 & $\ldots$ & $\ldots$ & D7 \\
\hline$\underline{1243507}$ & $\mathrm{C}$ & Unknown & Unknown & $\ldots$ & D2 & D5 & D7 \\
\hline$\underline{B G 065706}$ & $\mathrm{C}$ & Rabl3 & RAB, member of RAS oncogene family-like 3 & 16 & $\ldots$ & $\ldots$ & D7 \\
\hline BG078879 & C & Cox4il & Cytochrome c oxidase, subunit IVa & 8 & D2 & $\ldots$ & $\ldots$ \\
\hline$\underline{B G 088493}$ & $\mathrm{C}$ & Ptplad I & Protein tyrosine phosphatase-like A domain containing I & 9 & $\ldots$ & $\ldots$ & D7 \\
\hline$\underline{1378435}$ & C & Acad8 & acyl-Coenzyme A dehydrogenase family, member 8 & 9 & D2 & $\ldots$ & $\ldots$ \\
\hline$\underline{1429286}$ & C & 1600002K03Rik & RIKEN cDNA $1600002 \mathrm{~K} 03$ gene & 10 & $\ldots$ & D5 & $\ldots$ \\
\hline BG078872 & C & Pfkfb2 & 6-phosphofructo-2-kinase/fructose-2,6-biphosphatase 2 & 1 & $\ldots$ & D5 & $\ldots$ \\
\hline$\underline{420477}$ & $\mathrm{C}$ & Coll3al & Procollagen, type XIII, alpha I & 10 & D2 & $\ldots$ & $\ldots$ \\
\hline$\underline{402631}$ & C & Crebll & cAMP responsive element binding protein-like I & 17 & $\ldots$ & D5 & $\ldots$ \\
\hline$\underline{1445700}$ & C & 4930506M07Rik & RIKEN cDNA 4930506M07 gene & 19 & $\mathrm{D} 2 \mathrm{e}^{\mathrm{e}}$ & $\ldots$ & D7 \\
\hline$\underline{575274}$ & C & $\mathrm{Nvl}$ & Nuclear VCP-like & 1 & D2 & $\ldots$ & D7 \\
\hline$\underline{574369}$ & C & $\| 4$ & Interleukin 4 & 11 & D2 & D5 & D7 \\
\hline$\underline{B G 07 \mid 5 I I}$ & C & SuclgI & Succinate-CoA ligase, GDP-forming, alpha subunit & 6 & D2 & $\ldots$ & D7 \\
\hline$\underline{573178}$ & $\mathrm{C}$ & lgfbp7 & Insulin-like growth factor binding protein 7 & 5 & $\ldots$ & D5 & $\ldots$ \\
\hline$\underline{574126}$ & C & Dnaja3 & DnaJ (Hsp40) homolog, subfamily A, member 3 & 16 & D2 & $\ldots$ & D7 \\
\hline$\underline{1226596}$ & C & Femlc & fem-I homolog c (C.elegans) & 18 & $\mathrm{D} 2^{\mathrm{e}}$ & D5 & D7 \\
\hline$\underline{B G 087 / 53}$ & C & Bckdha & Branched chain ketoacid dehydrogenase EI, alpha polypeptide & 7 & $\mathrm{D} 2 \mathrm{e}$ & D5 & D7 \\
\hline$\underline{402019}$ & C & Golga7 & Golgi autoantigen, golgin subfamily a, 7 & 8 & $\mathrm{D} 2 \mathrm{e}^{\mathrm{e}}$ & $\ldots$ & D7 \\
\hline$\underline{574168}$ & C & Prosc & Proline synthetase co-transcribed & 8 & $\mathrm{D} 2 \mathrm{e}^{\mathrm{e}}$ & D5 & D7 \\
\hline BG073436 & C & Atp5b & ATP synthase, $\mathrm{H}+$ transporting mitochondrial FI complex, beta subunit & 10 & $\mathrm{D} 2^{\mathrm{e}}$ & D5 & D7 \\
\hline$\underline{574027}$ & C & MedI9 & Mediator of RNA polymerase II transcription, subunit 19 homolog (yeast) & 2 & D2 & D5 & $\ldots$ \\
\hline$\underline{1243989}$ & $\mathrm{C}$ & Mfhas I & Malignant fibrous histiocytoma amplified sequence I & 8 & D2 & D5 & D7 \\
\hline$\underline{373233}$ & $\mathrm{C}$ & Itgb5 & Integrin beta 5 & 16 & $\ldots$ & $\ldots$ & D7 \\
\hline$\underline{B G 085816}$ & $\mathrm{C}$ & Spna2 & Alpha-spectrin 2, brain & 2 & $\ldots$ & $\ldots$ & D7 \\
\hline$\underline{1446130}$ & $\mathrm{C}$ & 2410166105 Rik & RIKEN cDNA 24I0166105 gene & 4 & D2 & $\ldots$ & D7 \\
\hline$\underline{1263575}$ & $\mathrm{C}$ & Stard4 & StAR-related lipid transfer (START) domain containing 4 & 18 & D2 & D5 & $\ldots$ \\
\hline$\underline{1852943}$ & C & AnkrdI7 & Gene trap ankyrin repeat & 5 & $\ldots$ & $\ldots$ & D7 \\
\hline$\underline{389112}$ & C & Palm & Paralemmin & 10 & $\ldots$ & $\ldots$ & D7 \\
\hline$\underline{619816}$ & $\mathrm{C}$ & Smad5 & MAD homolog 5 (Drosophila) & 13 & $\ldots$ & $\ldots$ & D7 \\
\hline$\underline{641790}$ & C & $\mathrm{BC} 023814$ & cDNA sequence $B C 0238 / 4$ & 3 & $\ldots$ & $\ldots$ & D7 \\
\hline$\underline{1329743}$ & C & S3-12 & Plasma membrane associated protein, S3-12 & 17 & $\ldots$ & $\ldots$ & D7 \\
\hline$\underline{653795}$ & C & II4ra & Interleukin 4 receptor, alpha & 7 & D2 & $\ldots$ & D7 \\
\hline$\underline{640435}$ & $\mathrm{C}$ & lgsf3 & Immunoglobulin superfamily, member 3 & 3 & $\ldots$ & $\ldots$ & D7 \\
\hline$\underline{387990}$ & $\mathrm{C}$ & 0610040D20Rik & RIKEN cDNA 0610040D20 gene & 9 & D2 & D5 & $\ldots$ \\
\hline BG075962 & $\mathrm{C}$ & Peci & Peroxisomal delta3, delta2-enoyl-Coenzyme $\mathrm{A}$ isomerase & 13 & D2 & $\ldots$ & $\ldots$ \\
\hline BG086412 & $\mathrm{C}$ & Rpl3 I & Ribosomal protein L3I & 1 & D2 & $\ldots$ & $\ldots$ \\
\hline BG075914 & C & Rps9 & Ribosomal protein S9 & 7 & D2 & $\ldots$ & $\ldots$ \\
\hline$\underline{639852}$ & C & Bsg & Basigin & 10 & D2 & $\ldots$ & $\ldots$ \\
\hline
\end{tabular}


Table 2: CM-specific genes obtained by use of the Welch $\mathbf{t}$ test and a Bonferroni correction (Continued)

\begin{tabular}{|c|c|c|c|c|c|c|c|}
\hline$\underline{573106}$ & $\mathrm{C}$ & Slc25al & Solute carrier family 25 (mitochondrial carrier; citrate transporter), member I & 16 & D2 & $\ldots$ & D7 \\
\hline$\underline{574123}$ & C & Suds3 & Suppressor of defective silencing 3 homolog (S. cerevisiae) & 5 & D2 & $\ldots$ & $\ldots$ \\
\hline BG088819 & C & I8I0043G02Rik & RIKEN cDNA I8I0043G02 gene & 10 & D2 & $\ldots$ & $\ldots$ \\
\hline BG088858 & C & 1810044A24Rik & RIKEN cDNA 18I0044A24 gene & 15 & D2 & D5 & D7 \\
\hline BG078275 & C & C230096ClORik & RIKEN cDNA C230096CI0 gene & 4 & D2 & $\ldots$ & D7 \\
\hline$\underline{573120}$ & $\mathrm{C}$ & $2310035 \mathrm{~K} 24 \mathrm{Rik}$ & RIKEN cDNA $2310035 \mathrm{~K} 24$ gene & 2 & D2 & D5 & $\ldots$ \\
\hline BG076791 & C & Eefld & Eukaryotic translation elongation factor I delta (guanine nucleotide exchange protein) & 15 & D2 & $\ldots$ & $\ldots$ \\
\hline BG087946 & $\mathrm{C}$ & Ubtf & Transcription factor UBF & 11 & D2 & D5 & D7 \\
\hline$\underline{372316}$ & $\mathrm{C}$ & Ly6h & Lymphocyte antigen 6 complex, locus $\mathrm{H}$ & 15 & D2 & $\ldots$ & D7 \\
\hline$\underline{1243611}$ & C & Tmem23 & Transmembrane protein 23 & 19 & D2 & D5 & D7 \\
\hline$\underline{574299}$ & $\mathrm{C}$ & 2700059D2 I Rik & RIKEN cDNA 2700059D2I gene & 4 & $\ldots$ & D5 & D7 \\
\hline BG064820 & $\mathrm{C}$ & Sael & SUMOI activating enzyme subunit I & 7 & D2 & $\ldots$ & $\ldots$ \\
\hline BG07/827 & C & Ppmlb & Protein phosphatase IB, magnesium dependent, beta isoform & 17 & D2 & $D 5^{f}$ & $\ldots$ \\
\hline BG086606 & $\mathrm{C}$ & Txndc 12 & Thioredoxin domain containing 12 (endoplasmic reticulum) & 4 & D2 & $\ldots$ & $\ldots$ \\
\hline BG085962 & C & Gsn & Gelsolin & 2 & D2 & $\ldots$ & $\ldots$ \\
\hline$\underline{575308}$ & $\mathrm{C}$ & Dus3l & Dihydrouridine synthase 3-like (S. cerevisiae) & 17 & $\ldots$ & $\ldots$ & D7 \\
\hline$\underline{573228}$ & $\mathrm{C}$ & Fycol & FYVE and coiled-coil domain containing I & 9 & D2 & D5 & $\ldots$ \\
\hline BG08I532 & $\mathrm{C}$ & Sfpq & Splicing factor proline/glutamine rich (polypyrimidine tract binding protein associated) & 4 & D2 & $\mathrm{D}^{\mathrm{f}}$ & $D 7^{f}$ \\
\hline BG0726|4 & C & Rps 16 & Ribosomal protein S16 & 7 & D2 & $\ldots$ & $\ldots$ \\
\hline BG078928 & C & Stmn3 & Stathmin-like 3 & 2 & $\ldots$ & $\ldots$ & D7 \\
\hline$\underline{1226306}$ & C & $\mathrm{Cd} 274$ & CD274 antigen & 19 & D2 & D5 & $\ldots$ \\
\hline$\frac{\text { AW54462 }}{\underline{8}}$ & $\mathrm{C}$ & Itgb | & Integrin beta I (fibronectin receptor beta) & 8 & D2 & $\ldots$ & D7 \\
\hline BG087322 & $\mathrm{C}$ & Rps6ka2 & Ribosomal protein S6 kinase, 90 kD, polypeptide 2 & 17 & D2 & D5 & $\ldots$ \\
\hline$\underline{641964}$ & $\mathrm{C}$ & $\mathrm{Cd} 4$ & CD4 antigen & 6 & D2 & D5 & $\ldots$ \\
\hline BG07/505 & $\mathrm{C}$ & SIc28a3 & Solute carrier family 28 (sodium-coupled nucleoside transporter), member 3 & 13 & D2 & D5 & $\ldots$ \\
\hline BG088775 & $\mathrm{C}$ & Znhit3 & Zinc finger, HIT type 3 & 11 & D2 & D5 & $\ldots$ \\
\hline BG069977 & $\mathrm{C}$ & Pmml & Phosphomannomutase I & 15 & D2 & D5 & $\ldots$ \\
\hline$\underline{574022}$ & $\mathrm{E}$ & I2000I5MI2Rik & RIKEN cDNA I2000I5MI 2 gene & 3 & D2 & $\ldots$ & $\ldots$ \\
\hline BG086286 & E & Cirbp & Cold inducible RNA binding protein & 10 & D2 & $\ldots$ & $\ldots$ \\
\hline$\underline{598493}$ & $\mathrm{E}$ & Ifit3 & Interferon-induced protein with tetratricopeptide repeats 3 & 19 & D2 & D5 & $\ldots$ \\
\hline$\underline{617022}$ & " & " & " & " & " & $"$ & " \\
\hline$\underline{1329893}$ & $\mathrm{E}$ & 26000 IOEOIRik & RIKEN cDNA 26000 I0EOI gene & 2 & $\ldots$ & $\cdots$ & D7 \\
\hline BG076479 & E & Ctla2a & Cytotoxic T lymphocyte-associated protein 2 alpha & 13 & $\ldots$ & $D 5^{f}$ & D7 \\
\hline BG088327 & $\mathrm{E}$ & Sf3bl & Splicing factor 3b, subunit I, I55 kDa & 1 & $\ldots$ & D5 & $\ldots$ \\
\hline BG076355 & E & Stat3 & Signal transducer and activator of transcription 3 & 11 & D2 & $\ldots$ & $\ldots$ \\
\hline$\underline{643048}$ & $\mathrm{E}$ & Gbp6 & Guanylate binding protein 6 & 3 & $\ldots$ & D5 & $\ldots$ \\
\hline$\underline{638199}$ & E & $\mathrm{H} 2-\mathrm{T} 22$ & Histocompatibility 2, T region locus 22 & 17 & D2 & $D 5^{f}$ & $\ldots$ \\
\hline$\underline{638232}$ & E & Jundm2 & Jun dimerization protein 2 & 12 & D2 & D5 & $\cdots$ \\
\hline$\underline{1394984}$ & E & Rps I4 & Ribosomal protein SI4 & 18 & $\ldots$ & $\ldots$ & D7 \\
\hline
\end{tabular}

a Clone ID or GenBank accession no.

b The genes II4ra and Ifit3 were duplicates (the same clone for the gene II4ra and two different clones for the gene Ifit3). Thus, there were I77 genes identified.

c Chromosomal localization.

d The days for which the Welch $t$ test was significant $(<0.000 \mathrm{I})$ after Bonferroni correction are mentioned.

e Significant difference was observed only between BALB/c and C57BL/6.

f Significant difference was observed only between $B A L B / c$ and $C B A /]$.

fied a number of genes differentially regulated between CM-R and CM-S mice. The 327 most differentially expressed genes identified by the SAM analysis allowed the complete discrimination between CM-R and CM-S mice according to the time of infection. The same result was obtained with the subset of 177 genes identified by the Welch t test (data not shown). This further confirms our previous study that investigated gene expression at the time of CM onset [8].

EASE analysis of either the 327 genes or the 177 genes revealed that some of the most represented biological process categories were related to the "defense response", such as the "response to parasite" or the "inflammatory response" terms. This was further supported by the results of the KEGG pathway analysis. In addition, genes were found to be involved in KEGG pathways related to metab- olism, such as "oxidative phosphorylation", "glycolysis/ gluconeogenesis", or "tryptophan metabolism". The analysis of functional annotation also revealed GO terms and KEGG pathways related to brain, such as the "axon guidance" and the "neurodegenerative disorders" KEGG pathways.

Overall, the analysis of functional annotation is consistent with the view that mouse CM is characterized among others by the deregulation of both immune response and glucose metabolism $[11,12]$. This leads to an abnormal increase in the inflammatory response and to hypoglycemia and acidosis in CM-S mice $[11,12]$. In addition, our data provide evidence of an intrinsic deficiency in oxidative phosphorylation, and the functional annotations related to brain disease suggest the role of genes expressed by brain cells in resistance or susceptibility to CM. Thus, 
Table 3: Functional KEGG annotation of genes associated with resistance or susceptibility to $\mathrm{CM}$

\begin{tabular}{lc}
\hline KEGG pathways & $\begin{array}{c}\text { Percentage of } \\
\text { significant CM genes }\end{array}$ \\
\hline Ribosome & 13.3 \\
Cell adhesion molecules (CAMs) & 10.0 \\
Antigen processing and presentation & 8.3 \\
Oxidative phosphorylation & 6.7 \\
Hematopoietic cell lineage & 6.7 \\
Leukocyte transendothelial migration & 6.7 \\
ECM-receptor interaction & 6.7 \\
Cytokine-cytokine receptor interaction & 5.0 \\
Glycolysis/Gluconeogenesis & 5.0 \\
Fructose and mannose metabolism & 5.0 \\
Regulation of actin cytoskeleton & 5.0 \\
T cell receptor signaling pathway & 5.0 \\
Jak-STAT signalling pathway & 5.0 \\
Axon guidance & 5.0 \\
Valine, leucine and isoleucine degradation & 5.0 \\
Focal adhesion & 5.0 \\
Tight junction & 5.0 \\
MAPK signaling pathway & 3.3 \\
B cell receptor signaling pathway & 3.3 \\
Tryptophan metabolism & 3.3 \\
Propanoate metabolism & 3.3 \\
Butanoate metabolism & 3.3 \\
Bile acid biosynthesis & 3.3 \\
Fatty acid metabolism & 3.3 \\
Adipocytokine signaling pathway & 3.3 \\
Neuroactive ligand-receptor interaction & 3.3 \\
Neurodegenerative Disorders & 3.3 \\
& \\
\hline
\end{tabular}

a Only the KEGG pathways that contained at least two genes were represented.

b Of the 177 significant CM genes, 60 genes were annotated.

despite a number of genes identified by our microarray analysis, which may highlight highly complex interactions between the parasite and the host, several major features of the transcriptional profile can be deduced.
A

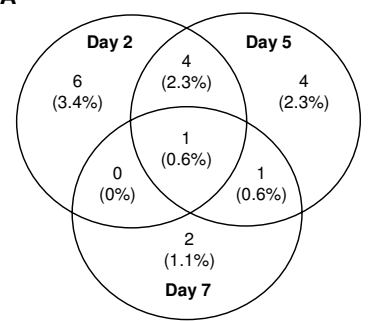

B

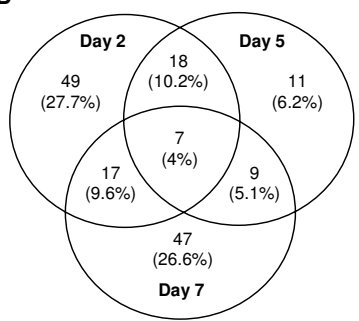

Figure 6

Genes differentially expressed between CM-R and CM-S mice on days 2,5 , and 7 . The number of genes upregulated in CM-S mice compared to CM-R mice $(\mathbf{A})$, and the number of genes up-regulated in CM-R mice compared to CM-S mice (B) are shown.
First, $\mathrm{PbA}$ infection affects the expression of genes involved in metabolic energy pathways. The expression of Uqcrq, Cox4i1, Ndufb8, Atp6v1g2 and Atp5b involved in oxidative phosphorylation was upregulated in CM-R mice on day 2 post-infection [see Additional file 2], suggesting that cerebral oxidative metabolism may be stimulated by $\mathrm{PbA}$ infection in CM-R mice. In contrast, these genes were downregulated in C57BL/6 mice on day 2 post-infection and in CBA/J mice on day 7 post-infection, while only $\mathrm{Ndufb8}$ was downregulated in CBA/J mice on day 2 postinfection. These transcriptional changes were associated with CM. A similar pattern was observed for Hmgcs1 involved in ketone metabolism. In the same way, lower $\mathrm{NAD}+$ /NADH levels and decreased mitochondrial function have been observed in CM-S mice by others $[12,13]$. These observations may be related to hypoxia and hypoglycemia, which reflect the low level of metabolic energy substrates. Alternatively, our data are consistent with the "cytopathic hypoxia" hypothesis, which rather proposes an adequate oxygen supply but an abnormal oxygen use [14]. Also, the low level of oxidative phosphorylation gene expression and ketone bodies pathway genes that we detected in the brain of CM-S mice suggests that cerebral oxidative metabolism may be inhibited even without oxygen delivery being impaired.

This metabolic disturbance also leads to lactate production and acidosis. In addition, this leads to an accumulation of ADP, which favors platelet aggregation [15]. Interestingly, platelet aggregation is known to be stimulated by PAF acether and inhibited by AMPc, the expression of which is inhibited by LIS1 (PAFAH1B1) and PDE4B, respectively $[16,17]$. Overall, the lower expression of Pafah1b1 in CM-S mice compared to CM-R mice [8], the higher expression of $P d e 4 b$ in CM-S mice compared to $\mathrm{CM}-\mathrm{R}$ mice, and the metabolic disturbance leading to an accumulation of ADP may participate in the platelet aggregation process in the cerebral microvasculature of CM-S mice.

Second, it is likely that the inflammatory response plays a major role in CM pathogenesis. In particular, a surge of IFN $\gamma$ production at 3 to 4 days p.i. was demonstrated to be essential for murine $\mathrm{CM}$, and this may be due to the absence of regulation in IFN $\gamma$ pathways at early stages in $\mathrm{PbA}$ infection [18-20]. IFN $\gamma$ is a proinflammatory cytokine typically produced by Th1 lymphocytes, and it is thought that the Th2 response protects from CM [11]. In this way, our microarray analysis showed that IL4 and $I L 4 R$ were over-expressed in CM-R mice from day 2. Similarly, Dnaja3, Foxo3a, and Ptpns1 that inhibit the activity of NF-kB [21-23] had lower expression levels in CM-S mice than in CM-R mice, while Nfkbia, a marker of the NF$\mathrm{kB}$ signalling pathway involved in inflammation [24], was over-expressed in CM-S mice. In addition, C1qa and Pde $4 b$ 
Table 4: Protein expression in brain of CM-R and CM-S mice upon malaria infection

\begin{tabular}{|c|c|c|c|c|c|c|c|c|c|}
\hline & \multicolumn{3}{|c|}{$\mathrm{d} 0 / \mathrm{d} 2$} & \multicolumn{3}{|c|}{ D5 } & \multicolumn{3}{|c|}{ d7 } \\
\hline & $\mathrm{BALB} / \mathrm{c}$ & $\mathrm{CBA} / \mathrm{J}$ & C57BL/6 & $\mathrm{BALB} / \mathrm{c}$ & $\mathrm{CBA} / \mathrm{J}$ & C57BL/6 & $\mathrm{BALB} / \mathrm{c}$ & $\mathrm{CBA} / \mathrm{J}$ & C57BL/6 \\
\hline Reelin & - & - & - & ++ & - & - & +++ & - & - \\
\hline$\beta$-Amyloid & - & - & - & - & + & + & - & ++ & ++ \\
\hline
\end{tabular}

$\mathrm{n}=3$, at least 25 brain fields analysed per mouse.

-: no positive vessel

+ : from I to 5 postive areas/vessels per field

++ : from 6 to 10 postive areas/vessels per field

+++ : $>10$ positive areas/vessels per field

$\mathrm{d} 0 / \mathrm{d} 2$ : results were similar for both day 0 and day 2 .

that are involved in the inflammatory response $[17,25]$ were found to be over-expressed in CM-S mice compared to CM-R mice. S100a10 that inhibits the activity of phospholipase A2 [26], and Bcl6 that inhibits the production of MIP-alpha and IP-10 [27] were under-expressed in CM$\mathrm{S}$ mice compared to CM-R mice. Blood cells are known to be involved in the inflammatory response due to malarial infection. Indeed, CD4+ and CD8+ T lymphocytes, platelets, monocytes have been shown to cooperate in the cerebral microvasculature, and this causes inflammation, endothelial cell damage, and hemorrhages [9,20,28-30]. In addition, Gzmb, whose expression was up-regulated in CM-S mice [8], encodes granzyme B in cytotoxic T lymphocytes, and is thought to be involved in the breakdown of the blood-brain barrier [31]. The influence of immune responses on the blood-brain barrier may be partly reflected by changes in expression of genes involved in either cytoskeletal and tight-junction pathways or cell adhesion pathways in CM-S mice.

It has been also suggested that glial cells actively participate in the local inflammatory response caused by malarial infection [32]. In this way, glial cells that have been shown to be activated on day 3 post-infection by PbA can produce $\mathrm{C} 1 \mathrm{q}$ components $[32,33]$. The NF-kB signalling pathway has been demonstrated in these cells [34]. Interestingly, $C d 200$ that is implicated in the control of the activation of glial cells was strongly under-expressed in the CBA/J CM-S mice [35]. Besides, the expression of IL4 and IL4R was shown to be higher in CM-R mice than in CM-S mice. Since IL-4 induces apoptosis in activated glial cells that express IL-4R [36], IL-4 may contribute to the down-regulation of brain inflammation in CM-R mice.

Third, genes involved in the neuroprotection/neurotoxicity balance and/or in neurogenesis may protect the host against CM. This hypothesis is based on changes in tryptophan metabolism caused by PbA infection. Sanni et al (1998) showed an increase of the activity of indoleamine 2,3-dioxygenase whose expression is induced by TNF and IFN [37]. This leads to an increase of the ratio quinolinic acid/kynurenic acid, and to neuro-excitotoxic damage associated with CM. In humans, high levels of quinolinic acid have been associated with CM [38]. Interestingly, genes involved in tryptophan metabolism, such as Ube3a, Prnt3, and Aldh1b1, were differentially regulated between $\mathrm{CM}-\mathrm{R}$ and CM-S mice. The expression of these genes was enhanced by infection in CM-R mice. In addition, genes having a neuroprotective role, such Agtr2, Bag1, Csnk1a1 and Reln [39-42], were shown to be over-expressed in CM$\mathrm{R}$ mice compared to CM-S mice. Indeed, Rtn3 and Creb1 that are markers of neuronal survival $[43,44]$, were also over-expressed in CM-R mice. Besides, Reln and Dab1, which were over-expressed in CM-R mice, are known to be involved in neurogenesis $[45,46]$. Similarly, Pafah 1 b1 whose expression was associated with resistance to CM [8] is involved in the Reln pathway [46]. This suggests that $\mathrm{CM}-\mathrm{S}$ mice are deficient in neurogenesis, and that they cannot repair neuronal damages. In contrast, CM-R mice might be able to repair such damage (Figure 7).

So far, little research has been conducted on the issue of neuroprotective responses in CM. Interestingly, the Reln pathway that inhibits the phosphorylation of the protein Tau has been recently proposed as a protective mechanism against Alzheimer'disease [47]. In addition, decreased ribosomal RNA levels and decreased rates for protein synthesis have been recently described in Alzheimer'disease [48], while we showed the down-regulation of several genes encoding ribosomal proteins in CM-S mice. In the same way, Medana et al (2002) detected $\beta$ amyloid precursor protein in humans with CM [49], and we report here the $\beta$-amyloid protein in brains of CM-S mice but not in CM-R mice. This result is consistent with the down-regulation of Arc, Itm2b, Bsg, Rtn3, and Il4 in CM-S mice, and with the up-regulation of Pde $4 b$ in CM-S mice. Indeed, Itm $2 b, B s g, R t n 3$, and Il4 inhibit the production of the $\beta$-amyloid protein [50-53]. Besides, the $\beta$-amyloid protein inhibits the expression of Arc, while it increases the expression of Pde $4 b$ [25,54]. These observations suggest that cerebral malaria and Alzheimer'disease share some common mechanisms of pathogenesis. 


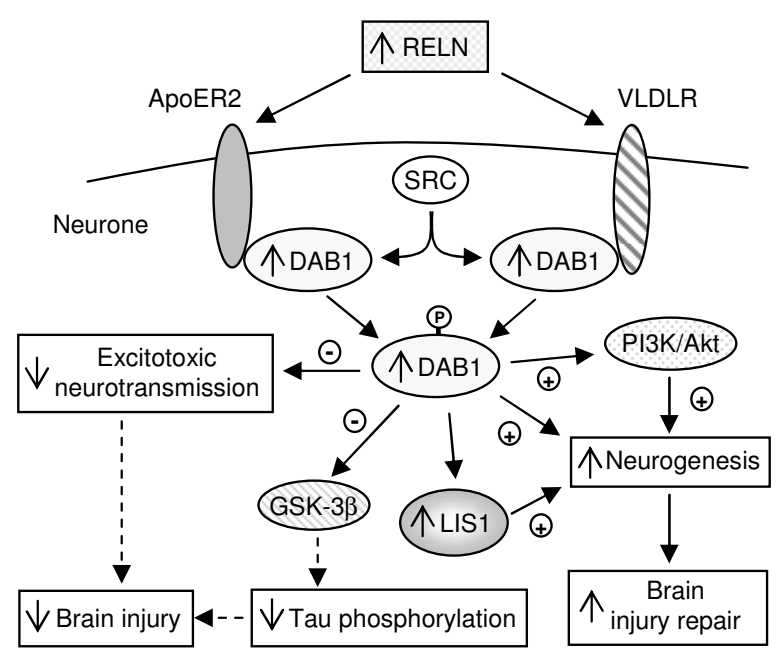

Figure 7

Schematic diagram showing the possible effects of the reelin pathway in protection from $\mathbf{C M}$. Reelin (RELN) is an extracellular matrix serine protease expressed in some neurons, such as GABAergic interneurons, which inhibit excitotoxic neurotransmission [45]. RELN that is secreted into the extracellular space acts by paracrine and autocrine mechanisms. RELN interacts with very low-density lipoprotein receptors (VLDLR) and apolipoprotein E type 2 receptors (ApoER2) leading to tyrosine phosphorylation of the adaptor protein Disabled-I (DABI) by the SRC family kinases (SRC) [42]. DABI activation, in turn, activates PI3K/ Akt signalling, which has been implicated in neuronal migration during development and adulthood. In addition, phosphorylated DABI interacts with LISI, a protein encoded by Pafah $/ b /$, which associates with microtubules and modulates neuronal migration [46]. LISI may be required for regulating crucial steps of reelin-dependent neuronal positioning. In parallel, phosphorylated DABI inhibits glycogen synthase kinase $3 \beta$ (GSK3 $\beta$ ), a kinase known to phosphorylate Tau protein at multiple sites. Therefore, the activation of RELN pathway diminishes the level of hyperphosphorylated Tau protein, which is a biomarker of brain injury. In particular, hyperphosphorylated Tau protein is a component of the neurofibrillary tangles involved in Alzheimer's disease. Reln, Dab I and Pafah /b/ were shown to be over-expressed in CM-R mice compared to CM-S mice. The activation of RELN signalling may inhibit excitotoxic neurotransmission and Tau phosphorylation, and may activate neurogenesis in CM-R mice. This may lead to diminished brain injury and to increased brain injury repair. Solid arrows represent influences on the activity of proteins or physiological mechanisms. Dashed arrows represent impaired effects on the activity of proteins or physiological mechanisms. Negative signs indicate inhibition, and positive signs indicate activation.

\section{Conclusion}

In this study, we have confirmed that gene-expression profiling discriminates between CM-R and CM-S mice, and we have identified genes whose expression showed consistent differential expression between CM-R and CM$S$ mice at early and late stages of infection. The analysis of gene functional annotation reveals several major features. First, it indicated that brain metabolic energy metabolism was early and deeply disturbed in CM-S mice, suggesting that high lactate production may be due rather to metabolic disturbance than to deficient oxygen supply. Second, the influence of inflammatory response on CM was also clearly detected, and our data are consistent with an active role of microglial cells in local inflammation. Third, the outcome of infection may critically depend on either cerebral tissue protective responses or brain repair capacity. Overall, our microarray analysis may give a global overview of critical events occurring in CM-S mice compared to CM-R mice. Searching for polymorphisms that alter the expression of genes identified should help in determining the genetic control of cerebral malaria. This analysis also revealed some promising areas for exploration that may both provide new insight into the key events that govern $\mathrm{CM}$ pathogenesis and the development of therapeutic strategies. In particular, novel neuroprotective therapies may be proposed as adjuncts to anti-malarial therapy.

\section{Methods}

\section{Mouse strains and phenotyping}

Six to 8 weeks old BALB/C (CM-R strain), C57BL/6J and $\mathrm{CBA} / \mathrm{J}$ (CM-S strains) female, were obtained from IFFA CREDO (Ch. River Lab, France) and kept in our facilities. Three mice from each strain were not infected, and 22 BALB/c, 17 C57BL/6J, and $15 \mathrm{CBA} / \mathrm{J}$ were infected by i.p. injection of $10^{6} \mathrm{PbA}$ parasitized erythrocytes. The frozen stabilates were obtained with an uncloned line, and were prepared from CBA/J day 7 -infected mice [30]. The parasite was conserved as stabilates of $10^{7}$ parasitized erythrocytes stored in liquid nitrogen in Alsever's solution containing $10 \%$ glycerol. Parasitemia was monitored daily by blood smear. No difference was observed between mouse strains. Parasitemia was $0.8 \% \pm 0.9$, $4.5 \% \pm 2.2$, and $8.7 \% \pm 5.3$ on days 2,5 , and 7 post-infection, respectively. The CM-S mice developed a neurological syndrome (mono-, hemi-, para-, or tetraplegia, ataxia, deviation of the head, and convulsions), which occurred 6 to 7 days after parasite inoculation with a cumulative mortality of $100 \%$. The CM-R mice did not present neurologic lesions and died during the $3^{\text {rd }}$ or the $4^{\text {th }}$ week of infection, with severe anaemia and hyperparasitemia [9]. The parasitemia of CM-R mice was $16.6 \% \pm 8.9$ and $62.8 \% \pm 25.7$ on days 9 and 15 , respectively. 


\section{Organ sampling}

Brains were taken from CM-R and CM-S mice before and after infection. Brains from three uninfected mice were taken for each strain. Three, 4, and $6 \mathrm{CBA} / \mathrm{J}$ were analyzed on days 2, 5, and 7 post-infection, respectively, while 4, 4, and $9 \mathrm{C} 57 \mathrm{BL} / 6$ were analyzed on days 2,5 , and 7 postinfection, respectively. Brains from 3, 4, 5, 5, and 5 BALB/ c mice were taken on days 2, 5, 7, 9 and 15 post-infection. Brains were completely removed and were cut into two parts: one part was frozen in RNALater (Qiagen, TM) until RNA analysis, and the other part was embedded in Tissue Tek (Leica), snap frozen in liquid nitrogen, and kept at $80^{\circ} \mathrm{C}$ until histopathological analysis of cryosections.

\section{Immunochemistry}

For immunostaining, frozen sections were incubated for 45 minutes with primary monoclonal antibodies directed against murine Reelin and $\beta$-Amyloid peptide (Santa Cruz, Tebu Bio) after saturation with appropriate serum. After washing, sections were incubated for 45 minutes with biotinylated polyclonal antibodies, followed by the addition of HRPO-avidin (anti-rat or anti-hamster ABC kits; Vector, Peterborough, England). Color reaction was obtained by the addition of Novared (AbCys). Slides were counterstained with Mayer's hematoxylin before permanent mounting with Entellan (Merck, Brussels, Belgium). Slides were pictured at 200 magnification using an Eclipse 800 microscope (Nikon, Champignysur- Marne, France) and digital camera; labelling was then analyzed by quantitative digitalized image analysis using Lucia software (Nikon). At least 3 brains were sampled for each time point and each mouse strain and image analysis was performed on an average of 25 microphotographs per mouse.

\section{RNA isolation and cDNA microarray hybridizations}

Total RNA from brains was extracted using TRIzol reagent (Gibco-BRL, Life Technologies). The quality of RNA was confirmed on a formaldehyde agarose gel, and the concentration of RNA was determined by reading absorbance at 260/280 nm. RNA from 2 CBA/J mice had an inadequate quality, and was not further processed. Each mRNA sample extracted from an individual brain was run on a single microarray. In addition, three samples were run on several microarrays, and were considered as technical replicates: samples from CBA/J, C57BL/6, and BALB/c mice were run on 5,2 , and 2 microarrays, respectively. All microarray procedures were done at our microarray core facility [55]. cDNA microarrays were designed and prepared as described [56]. Briefly, the microarrays used in this study were composed of 8388 sequences. The following cDNA libraries were used: the NIA Mouse $15 \mathrm{~K}$ cDNA clone set, 2NbMT (thymus), NbMLN (lymph node), and $3 \mathrm{NbMS}$ (spleen). Detailed descriptions of these cDNA libraries are available at the UniGene database website (2NbMT: Lib.544, 3NbMS: Lib.553, NbMLN: Lib.567,
NIA 15 K: Lib.8622) [57]. PCR amplification was performed as previously described [56], and PCR products were spotted onto nylon membranes (Hybond-N+, Amersham) with a MicroGrid II arrayer (Affymetrix, Santa Clara, CA). About 10\% of the genes included in this clone set are represented by two or more different cDNA clones, providing internal controls to assess the reproducibility of gene expression measurements. Microarrays were hybridized with ${ }^{33} \mathrm{P}$-labelled probes, first with an oligonucleotide sequence common to all spotted PCR products ( 5 'TCACACAGGAAACAGCTATGAC-3'), then after stripping, with complex probes made from $5 \mu \mathrm{g}$ of retrotranscribed total RNA. Probe preparations, hybridizations and washes were carried out as described previously [56]. After $48 \mathrm{~h}$ hybridization, arrays were scanned with a FUJI BAS5000 machine at $25 \mu \mathrm{m}$ resolution. Hybridization signals were quantified using ArrayGauge software (Fuji Ltd, Tokyo, Japan).

\section{Microarray data analysis}

All images were carefully inspected, and spots with overestimated intensities due to neighborhood effects were manually excluded. The data were filtered such that only spots with intensities that were two times greater than the median background in either microarray were used in the analysis, and the signal intensities were then corrected to take into account the amount of spotted DNA and the variability of experimental conditions, as described [58]. Of the 8388 spotted clones, we selected the clones that had detectable expression levels in at least $80 \%$ of the experiments ( $n=2012)$. Unsupervised hierarchical clustering investigated relationships between samples and relationships between genes. It was applied to data log-transformed and median-centred using the Cluster and TreeView programs (average linkage clustering using Pearson's correlation as similarity metric) [59].

Microarray data were statistically analyzed using the TIGR $\mathrm{MeV}$ (MultiExperiment Viewer) v3.1 software [60]. Figure 1 shows an outline of data analysis. A one-way ANOVA and SAM (Significant Analysis of Microarrays) procedures were applied to look for time-, strain-, and CM-R/CM-Sspecific variation in gene expression in the full data set. One-way ANOVA and Welch t-statistics were used to analyze gene expression changes upon infection for each mouse strain. The values on days 2, 5, 7, 9 and 15 postinfection were compared to values on day 0 before infection. To search for gene expression changes associated with CM, a multi-class SAM procedure and a Welch t test were performed on the $\log 2$ ratios of infected vs uninfected samples. For each gene, the level of gene expression in each sample taken from an infected mouse was divided by the median of gene expression levels in samples taken from three uninfected mice. This calculation was done for 
each mouse strain. Multiple test corrections were performed [61].

The Expression Analysis Systematic Explorer (EASE) was used to search for common biological themes within gene lists generated by our microarray analysis [62]. EASE assigns identified genes to Gene Ontology (GO) terms, and tests whether specific biological pathways were overrepresented within specific gene clusters. A score based on Fisher Exact test reports the probability that the prevalence of a particular theme within a cluster is due to chance alone given the prevalence of that theme in the population of all genes under study. In addition, we checked whether the genes were included in a KEGG pathway [63].

All data are MIAME compliant and have been loaded into ArrayExpress database [64]. The ArrayExpress accession number of this experiment is E-MEXP-1018.

\section{Abbreviations}

CM: Cerebral Malaria

CM-S: Cerebral Malaria-Susceptible mice

CM-R: Cerebral Malaria-Resistant mice

PbA: Plasmodium berghei ANKA

GO: Gene Ontology

SAM: Significant Analysis of Microarrays

RELN: Reelin

\section{Competing interests}

The author(s) declare that they have no competing interests.

\section{Authors' contributions}

NFD participated in the design of the study, the injection of parasitized erythrocytes, the sample preparation, and the analysis of the data, carried out microarray experiments, and prepared the figures. NC participated in sample preparation and carried out imunochemistry experiments. DP, FJ, and $\mathrm{CN}$ designed and produced the microarrays. DP participated in the statistical analysis, and $\mathrm{CN}$ contributed to study design. MB participated in microarray hybridizations. PB and GG participated in the interpretation of data that concerned genes involved in the inflammatory response. NFD and PR carried out a systematic analysis of the functional annotation of genes identified. FAI and GG participated in the design of animal studies. PR conceived and coordinated the study, and wrote the manuscript. All authors read and approved the final manuscript.

\section{Additional material}

\section{Additional File 1}

The full list of the 327 genes that discriminated between early and late infection stages, between mouse strains, and between CM-R and CM-S mice.

Click here for file

[http://www.biomedcentral.com/content/supplementary/1471-

2164-8-452-S1.xls]

\section{Additional File 2}

The graphical representation of expression profiles of genes involved in oxidative phosphorylation in CM-S and CM-R mice.

Click here for file

[http://www.biomedcentral.com/content/supplementary/14712164-8-452-S2.pdf]

\section{Acknowledgements}

We thank Béatrice Loriod and Geneviève Victorero for technical support and advice. We also thank Frédéric Foucault for helpful discussions, and Andrew Mitchell for critical reading of the manuscript. We acknowledge the technical support of Marseille-Nice genopole.

Financial support: French Ministry of Research and Technology (PAL+ Program); Fondation pour la Recherche Médicale; PACA Conseil Régional; Conseil Général des Bouches du Rhône. NFD was supported by a studentship from the Fondation pour la Recherche Médicale. MB and NC are supported by a studentship from the French Ministry of Research and Technology.

\section{References}

I. Kwiatkowski DP: How malaria has affected the human genome and what human genetics can teach us about malaria. Am J Hum Genet 2005, 77(2): I7I-192.

2. Fortin A, Stevenson MM, Gros P: Complex genetic control of susceptibility to malaria in mice. Genes Immun 2002, 3(4): $177-186$.

3. Sexton AC, Good RT, Hansen DS, D'Ombrain MC, Buckingham L, Simpson K, Schofield L: Transcriptional profiling reveals suppressed erythropoiesis, up-regulated glycolysis, and interferon-associated responses in murine malaria. J Infect Dis 2004, I89(7): 1245-1256.

4. Schaecher K, Kumar S, Yadava A, Vahey M, Ockenhouse CF: Genome-wide expression profiling in malaria infection reveals transcriptional changes associated with lethal and nonlethal outcomes. Infect Immun 2005, 73(9):609I-6100.

5. Lovegrove FE, Pena-Castillo L, Mohammad N, Liles WC, Hughes TR, Kain KC: Simultaneous host and parasite expression profiling identifies tissue-specific transcriptional programs associated with susceptibility or resistance to experimental cerebral malaria. BMC Genomics 2006, 7:295.

6. Ylostalo J, Randall AC, Myers TA, Metzger M, Krogstad DJ, Cogswell $\mathrm{FB}$ : Transcriptome profiles of host gene expression in a monkey model of human malaria. J Infect Dis 2005, 19 I (3):400-409.

7. Griffiths MJ, Shafi MJ, Popper SJ, Hemingway CA, Kortok MM, Wathen A, Rockett KA, Mott R, Levin M, Newton CR, Marsh K, Relman DA, Kwiatkowski DP: Genomewide analysis of the host response to malaria in Kenyan children. J Infect Dis 2005, 191(10):1599-1611.

8. Delahaye NF, Coltel N, Puthier D, Flori L, Houlgatte R, Iraqi FA, Nguyen C, Grau GE, Rihet P: Gene-Expression Profiling Discriminates between Cerebral Malaria (CM)-Susceptible Mice and CM-Resistant Mice. J Infect Dis 2006, I 93(2):3 I2-32I. 
9. Lou J, Lucas R, Grau GE: Pathogenesis of cerebral malaria: recent experimental data and possible applications for humans. Clin Microbiol Rev 200I, I 4(4):8I0-20, table of contents.

10. Engwerda CR, Mynott TL, Sawhney S, De Souza JB, Bickle QD, Kaye PM: Locally up-regulated lymphotoxin alpha, not systemic tumor necrosis factor alpha, is the principle mediator of murine cerebral malaria. J Exp Med 2002, I 95( I 0): |37|- | 377.

II. Hunt NH, Grau GE: Cytokines: accelerators and brakes in the pathogenesis of cerebral malaria. Trends Immunol 2003, 24(9):491-499.

12. Rae C, McQuillan JA, Parekh SB, Bubb WA, Weiser S, Balcar VJ, Hansen AM, Ball HJ, Hunt NH: Brain gene expression, metabolism, and bioenergetics: interrelationships in murine models of cerebral and noncerebral malaria. Faseb J 2004, I 8(3):499-5I0.

13. Sanni LA, Rae C, Maitland A, Stocker R, Hunt NH: Is ischemia involved in the pathogenesis of murine cerebral malaria? $\mathrm{Am}$ J Pathol 200 I, I 59(3): I |05-I | I2.

14. Fink MP: Bench-to-bedside review: Cytopathic hypoxia. Crit Care 2002, 6(6):491-499.

15. Kunapuli SP, Dorsam RT, Kim S, Quinton TM: Platelet purinergic receptors. Curr Opin Pharmacol 2003, 3(2): I75-I80.

16. Hattori M, Aoki J, Arai $\mathrm{H}$, Inoue K: PAF and PAF acetylhydrolase in the nervous system. J Lipid Mediat Cell Signal 1996, I4( I3):99- 102 .

17. Ariga M, Neitzert B, Nakae S, Mottin G, Bertrand C, Pruniaux MP, Jin $S L$, Conti $M$ : Nonredundant function of phosphodiesterases 4D and 4B in neutrophil recruitment to the site of inflammation. J Immunol 2004, I 73( I 2):753 I-7538.

18. Grau GE, Heremans H, Piguet PF, Pointaire P, Lambert PH, Billiau A, Vassalli $P$ : Monoclonal antibody against interferon gamma can prevent experimental cerebral malaria and its associated overproduction of tumor necrosis factor. Proc Natl Acad Sci U S A 1989, 86(14):5572-5574.

19. Mitchell AJ, Hansen AM, Hee L, Ball HJ, Potter SM, Walker JC, Hunt $\mathrm{NH}$ : Early cytokine production is associated with protection from murine cerebral malaria. Infect Immun 2005 , 73(9):5645-5653.

20. de Kossodo S, Grau GE: Profiles of cytokine production in relation with susceptibility to cerebral malaria. J Immunol 1993, I 5 | (9):48 | |-4820.

21. Cheng H, Cenciarelli C, Tao M, Parks WP, Cheng-Mayer C: HTLVI Tax-associated hTid-I, a human DnaJ protein, is a repressor of Ikappa B kinase beta subunit. J Biol Chem 2002 277(23):20605-20610.

22. Lin L, Hron JD, Peng SL: Regulation of NF-kappaB, Th activation, and autoinflammation by the forkhead transcription factor Foxo3a. Immunity 2004, 2 I (2):203-2I3.

23. Neznanov N, Neznanova L, Kondratov RV, Burdelya L, Kandel ES, O'Rourke DM, Ullrich A, Gudkov AV: Dominant negative form of signal-regulatory protein-alpha (SIRPalpha /SHPS-I) inhibits tumor necrosis factor-mediated apoptosis by activation of NF-kappa B. J Biol Chem 2003, 278(6):3809-38। 5.

24. Blais $V$, Rivest $S$ : Inhibitory action of nitric oxide on circulating tumor necrosis factor-induced NF-kappaB activity and COX-2 transcription in the endothelium of the brain capillaries. J Neuropathol Exp Neurol 200I, 60(9):893-905.

25. Sebastiani G, Morissette C, Lagace C, Boule M, Ouellette MJ, McLaughlin RW, Lacombe D, Gervais F, Tremblay P: The cAMPspecific phosphodiesterase 4B mediates Abeta-induced microglial activation. Neurobiol Aging 2006, 27(5):69l-70I.

26. Donato R: Functional roles of $\mathbf{S} 100$ proteins, calcium-binding proteins of the EF-hand type. Biochim Biophys Acta 1999, |450(3): $|9|-23 \mid$

27. Shaffer AL, Yu X, He Y, Boldrick J, Chan EP, Staudt LM: BCL-6 represses genes that function in lymphocyte differentiation, inflammation, and cell cycle control. Immunity 2000, I3(2): $199-212$.

28. Hermsen C, van de Wiel T, Mommers E, Sauerwein R, Eling W: Depletion of CD4+ or CD8+ T-cells prevents Plasmodium berghei induced cerebral malaria in end-stage disease. Parasitology 1997, I/4 Pt ( I):7-12.

29. Yanez DM, Manning DD, Cooley AJ, Weidanz WP, van der Heyde HC: Participation of lymphocyte subpopulations in the pathogenesis of experimental murine cerebral malaria. Immunol 1996, I57(4): I620-I624.
30. Grau GE, Piguet PF, Engers HD, Louis JA, Vassalli P, Lambert PH: L3T4+ T lymphocytes play a major role in the pathogenesis of murine cerebral malaria. J Immunol I986, I 37(7):2348-2354.

3I. Potter S, Chan-Ling T, Ball HJ, Mansour H, Mitchell A, Maluish L, Hunt $\mathrm{NH}$ : Perforin mediated apoptosis of cerebral microvascular endothelial cells during experimental cerebral malaria. Int J Parasitol 2006, 36(4):485-496.

32. Medana IM, Chaudhri G, Chan-Ling T, Hunt NH: Central nervous system in cerebral malaria: 'Innocent bystander' or active participant in the induction of immunopathology? Immunol Cell Biol 200I, 79(2): I0I-I20.

33. Lynch NJ, Willis CL, Nolan CC, Roscher S, Fowler MJ, Weihe E, Ray DE, Schwaeble WJ: Microglial activation and increased synthesis of complement component $\mathrm{Clq}$ precedes blood-brain barrier dysfunction in rats. Mol Immunol 2004, 40( I 0):709-7I6.

34. Patrizio M: Tumor necrosis factor reduces cAMP production in rat microglia. Glia 2004, 48(3):24|-249.

35. Neumann $\mathrm{H}$ : Control of glial immune function by neurons. Glia 200I, 36(2): I91-199.

36. Park KW, Lee DY, Joe EH, Kim SU, Jin BK: Neuroprotective role of microglia expressing interleukin-4. J Neurosci Res 2005, 8 I (3):397-402

37. Sanni LA, Thomas SR, Tattam BN, Moore DE, Chaudhri G, Stocker R, Hunt NH: Dramatic changes in oxidative tryptophan metabolism along the kynurenine pathway in experimental cerebral and noncerebral malaria. Am J Pathol | 998, I 52(2):6 I I-6I9.

38. Dobbie M, Crawley J, Waruiru C, Marsh K, Surtees R: Cerebrospinal fluid studies in children with cerebral malaria: an excitotoxic mechanism? Am J Trop Med Hyg 2000, 62(2):284-290.

39. Li J, Culman J, Hortnagl H, Zhao Y, Gerova N, Timm M, Blume A, Zimmermann M, Seidel K, Dirnagl U, Unger T: Angiotensin AT2 receptor protects against cerebral ischemia-induced neuronal injury. Faseb J 2005, 19(6):617-619.

40. Liman J, Ganesan S, Dohm CP, Krajewski S, Reed JC, Bahr M, Wouters FS, Kermer P: Interaction of BAGI and Hsp70 mediates neuroprotectivity and increases chaperone activity. Mol Cell Biol 2005, 25(9):37|5-3725.

41. Chergui K, Svenningsson P, Greengard P: Physiological role for casein kinase I in glutamatergic synaptic transmission. J Neurosci 2005, 25(28):660I-6609.

42. Herz J, Chen Y: Reelin, lipoprotein receptors and synaptic plasticity. Nat Rev Neurosci 2006, 7( I I ):850-859.

43. Di Scala F, Dupuis L, Gaiddon C, De Tapia M, Jokic N, Gonzalez de Aguilar JL, Raul JS, Ludes B, Loeffler JP: Tissue specificity and regulation of the $\mathbf{N}$-terminal diversity of reticulon 3 . Biochem J 2005, 385(Pt I): I 25-I34.

44. Mantamadiotis T, Lemberger T, Bleckmann SC, Kern H, Kretz O, Martin Villalba A, Tronche F, Kellendonk C, Gau D, Kapfhammer J, Otto C, Schmid W, Schutz G: Disruption of CREB function in brain leads to neurodegeneration. Nat Genet 2002, 3 I (I):47-54.

45. Won SJ, Kim SH, Xie L, Wang Y, Mao XO, Jin K, Greenberg DA: Reelin-deficient mice show impaired neurogenesis and increased stroke size. Exp Neurol 2006, I 98(I):250-259.

46. Assadi AH, Zhang G, Beffert U, McNeil RS, Renfro AL, Niu S, Quattrocchi CC, Antalffy BA, Sheldon M, Armstrong DD, Wynshaw-Boris A, Herz J, D'Arcangelo G, Clark GD: Interaction of reelin signaling and LisI in brain development. Nat Genet 2003, 35(3):270-276.

47. Deutsch SI, Rosse RB, Deutsch LH: Faulty regulation of tau phosphorylation by the reelin signal transduction pathway is a potential mechanism of pathogenesis and therapeutic target in Alzheimer's disease. Eur Neuropsychopharmacol 2006, I 6(8):547-55 I.

48. Ding Q, Markesbery WR, Chen Q, Li F, Keller JN: Ribosome dysfunction is an early event in Alzheimer's disease. I Neurosci 2005, 25(40):9171-9175.

49. Medana IM, Day NP, Hien TT, Mai NT, Bethell D, Phu NH, Farrar J Esiri MM, White NJ, Turner GD: Axonal injury in cerebral malaria. Am J Pathol 2002, I 60(2):655-666.

50. Matsuda S, Giliberto L, Matsuda Y, Davies P, McGowan E, Pickford F, Ghiso J, Frangione B, D'Adamio L: The familial dementia BRI2 gene binds the Alzheimer gene amyloid-beta precursor protein and inhibits amyloid-beta production. J Biol Chem 2005, 280(32):28912-28916.

5I. Zhou S, Zhou H, Walian PJ, Jap BK: CDI47 is a regulatory subunit of the gamma-secretase complex in Alzheimer's disease 
amyloid beta-peptide production. Proc Natl Acad Sci U S A 2005, I02(2I):7499-7504.

52. He W, Lu Y, Qahwash I, Hu XY, Chang A, Yan R: Reticulon family members modulate BACEI activity and amyloid-beta peptide generation. Nat Med 2004, 10(9):959-965.

53. Iribarren $P$, Chen $K$, Hu J, Zhang X, Gong W, Wang JM: IL-4 inhibits the expression of mouse formyl peptide receptor 2 , a receptor for amyloid betal-42, in TNF-alpha-activated microglia. J Immunol 2005, I 75(9):6100-6106.

54. Dickey CA, Gordon MN, Mason JE, Wilson NJ, Diamond DM, Guzowski JF, Morgan D: Amyloid suppresses induction of genes critical for memory consolidation in APP + PSI transgenic mice. J Neurochem 2004, 88(2):434-442.

55. TAGC website [http://tagc.univ-mrs.fr/]

56. Puthier D, Joly F, Irla M, Saade M, Victorero G, Loriod B, Nguyen C: A general survey of thymocyte differentiation by transcriptional analysis of knockout mouse models. J Immunol 2004 173(10):6109-6118.

57. UniGene database website - Library browser [http:// www.ncbi.nlm.nih.gov/UniGene/lbrowse2.cgi?TAXID 10090]

58. Bertucci F, Salas S, Eysteries S, Nasser V, Finetti P, Ginestier C Charafe-Jauffret E, Loriod B, Bachelart L, Montfort J, Victorero G, Viret F, Ollendorff V, Fert V, Giovaninni M, Delpero JR, Nguyen C Viens P, Monges G, Birnbaum D, Houlgatte R: Gene expression profiling of colon cancer by DNA microarrays and correlation with histoclinical parameters. Oncogene 2004, 23(7): |377-|39|.

59. Eisen MB, Spellman PT, Brown PO, Botstein D: Cluster analysis and display of genome-wide expression patterns. Proc Natl Acad Sci U S A 1998, 95(25): I4863-I 4868.

60. TIGR MeV (MultiExperiment Viewer) v3.I software [http:// www.tm4.org/mev.html]

61. Benjamini Y, Hochberg Y: Controlling the False Discovery Rate: a practical and powerful approach to multiple testing. J Royal Stat Soc Ser B 1995, 57(1):289-300.

62. Expression Analysis Systematic Explorer (EASE) [http:// david.abcc.ncifcrf.gov]

63. Kyoto Encyclopedia of Genes and Genomes (KEGG) [http:// www.genome.jp/kegg]

64. ArrayExpress database [http://www.ebi.ac.uk/arrayexpress]

Publish with Biomed Central and every scientist can read your work free of charge

"BioMed Central will be the most significant development for disseminating the results of biomedical research in our lifetime. "

Sir Paul Nurse, Cancer Research UK

Your research papers will be:

- available free of charge to the entire biomedical community

- peer reviewed and published immediately upon acceptance

- cited in PubMed and archived on PubMed Central

- yours - you keep the copyright

Submit your manuscript here:

http://www.biomedcentral.com/info/publishing_adv.asp
Biomedcentral 